\title{
Stability of the derivative of a canonical product
}

\author{
Matthias Langer, Harald WoraceK
}

\begin{abstract}
With each sequence $\alpha=\left(\alpha_{n}\right)_{n \in \mathbb{N}}$ of pairwise distinct and non-zero points which are such that the canonical product

$$
P_{\alpha}(z):=\lim _{r \rightarrow \infty} \prod_{\left|\alpha_{n}\right| \leq r}\left(1-\frac{z}{\alpha_{n}}\right)
$$

converges, the sequence

$$
\alpha^{\prime}:=\left(P_{\alpha}^{\prime}\left(\alpha_{n}\right)\right)_{n \in \mathbb{N}}
$$

is associated. We give conditions on the difference $\beta-\alpha$ of two sequences which ensure that $\beta^{\prime}$ and $\alpha^{\prime}$ are comparable in the sense that

$$
\exists c, C>0: \quad c\left|\alpha_{n}^{\prime}\right| \leq\left|\beta_{n}^{\prime}\right| \leq C\left|\alpha_{n}^{\prime}\right|, \quad n \in \mathbb{N} .
$$

The values $\alpha_{n}^{\prime}$ play an important role in various contexts. As a selection of applications we present: an inverse spectral problem, a class of entire functions and a continuation problem.
\end{abstract}

AMS MSC 2010: Primary 30D20; Secondary 30D15, 42A82, 34B20

Keywords: Canonical product, perturbation of zeros, inverse spectral problem, Krĕn class, positive definite function

\section{Introduction}

Our aim in the present paper is to show a stability result for the derivative of a canonical product evaluated at its zeros when the zeros of the product are perturbed. To illustrate this task, let us consider a toy example.

1.1 Example. Let $f$ be the entire function $f(z):=\frac{\sin (\pi z)}{\pi z}$. The sequence of zeros of $f$ is $+1,-1,+2,-2, \ldots$, which we denote by $\alpha=\left(\alpha_{n}\right)_{n \in \mathbb{N}}$. Then $f$ is given by the canonical product

$$
f(z)=\lim _{r \rightarrow \infty} \prod_{\left|\alpha_{n}\right| \leq r}\left(1-\frac{z}{\alpha_{n}}\right) .
$$

Let $\beta=\left(\beta_{n}\right)_{n \in \mathbb{N}}$ be a small perturbation of $\alpha$, say

$$
\beta_{n}-\alpha_{n}=\mathrm{O}\left(\frac{1}{n^{\varepsilon}}\right), \quad n \rightarrow \infty
$$


for some $\varepsilon>0$, and consider the corresponding canonical product (we tacitly assume that the numbers $\beta_{n}$ are pairwise distinct and non-zero):

$$
g(z):=\lim _{r \rightarrow \infty} \prod_{\left|\beta_{n}\right| \leq r}\left(1-\frac{z}{\beta_{n}}\right) .
$$

Then $g$ is an entire function of finite exponential type. The function $z g(z)$ is of sine type ${ }^{1}$, and hence the sequence $\left(\left|\beta_{n} g^{\prime}\left(\beta_{n}\right)\right|\right)_{n \in \mathbb{N}}$ is bounded from above and away from zero. The same is of course true for $\left(\left|\alpha_{n} g^{\prime}\left(\alpha_{n}\right)\right|\right)_{n \in \mathbb{N}}$. Since $\lim _{n \rightarrow \infty} \frac{\beta_{n}}{\alpha_{n}}=1$, we may say that

$$
\left|g^{\prime}\left(\beta_{n}\right)\right| \asymp\left|f^{\prime}\left(\alpha_{n}\right)\right| ;
$$

here and in the following we write $x_{n} \lesssim y_{n}$ if there exists a constant $c>0$ such that $x_{n} \leq c y_{n}$ for all $n \in \mathbb{N}$, and we write $x_{n} \asymp y_{n}$ if $x_{n} \lesssim y_{n}$ and $y_{n} \lesssim x_{n}$. Note that removing or adding a zero changes the asymptotics of $f^{\prime}\left(\alpha_{n}\right)$; so in some sense, the behaviour of $f^{\prime}\left(\alpha_{n}\right)$ depends sensitively on $\alpha_{n}$.

Now consider two arbitrary sequences $\alpha$ and $\beta$, only assuming that the products (1.1) and (1.3) converge. What conditions on the perturbation $\beta-\alpha$ ensure that (1.4) holds?

In our main result, Theorem 3.3, we give conditions which guarantee that (1.4) holds. They limit the size of the perturbation in two respects: (1) relative to the location of $\alpha_{n}$ and (2) relative to the regularity of the distribution of $\alpha$ measured by the separation

$$
s_{\alpha}(n):=\min \left\{\left|\alpha_{k}-\alpha_{n}\right|: k \neq n\right\}
$$

and by some sort of local density (see $r_{\alpha}$ in Definition 2.6 below). The rough picture is (as one would expect): if the sequence $\alpha$ is sparse and well-separated, large perturbations are allowed.

In Theorem 3.3 we do not assume any regularity of the sequence $\alpha^{2}$. For sequences that are regularly distributed the conditions of Theorem 3.3 can be weakened significantly. We make this precise in the two supplementary results Theorems 3.18 and 3.19; see also Corollary $3.20^{3}$.

The conditions in our theorems appear to be more or less sharp, cf. Remark 3.5. In order to construct examples to explicitly show this, one would have to deal with very irregularly distributed sequences, cf. Remark 3.15. To do explicit computations in such cases seems to be out of reach.

Our motivation to consider the question for stability of $\left|f^{\prime}\left(\alpha_{n}\right)\right|$ is that these quantities frequently appear in complex analysis and spectral theory. They have significance in a multitude of classical problems, e.g. weighted approximation problems like the Bernstein problem, power or trigonometric moment problems, extension theory of symmetric operators, spectral theory of canonical systems, etc. In such situations often the convergence of series of the form

$$
\sum_{n \in \mathbb{N}} \frac{c_{n}}{\left|f^{\prime}\left(\alpha_{n}\right)\right|^{\rho}}
$$

\footnotetext{
${ }^{1}$ For definition and theory of sine-type functions we refer to [L2, Lecture 22].

${ }^{2}$ Besides some weak condition in the extremal case that the product (1.1) has positive exponential type, cf. Remark 3.4.

${ }^{3}$ What we have observed in the above example is reobtained. In fact, Corollary 3.20 shows that one may even allow larger perturbations than in (1.2) (although then the function $z g(z)$ may not be of sine type anymore). See Example 3.22 for more details.
} 
with some $c_{n}>0$ and $\rho>0$ plays a role. Hence we can invoke our present results and deduce stability under small perturbations. To illustrate this vague principle, we discuss in the present paper three applications representing typical and descriptive instances:

- spectral functions of regular strings (Theorem 4.2);

- the Kreĭn class of entire functions (Theorem 4.4);

- continuations of a positive definite function on an interval (Theorem 4.8).

All those are classical topics. Only in the last example, we make a little excursion to the indefinite (Pontryagin space) world ${ }^{4}$.

Another interesting (and more serious) application of Theorem 3.3 is found in the theory of the Hamburger power moment problem. There, stability of $\mathrm{N}$-extremality and (in conjunction with Pontryagin space methods), stability of the index of determinacy of a measure can be studied. These results will be presented as part of the forthcoming work [LW2], [LW3].

The proof of Theorem 3.3 and its supplements is carried out by accurately estimating products and, for the case of regularily distributed sequences, using some facts about growth functions. We employ the standard procedure to split a canonical product into several parts and estimate each of them. However, it is not just "business as usual": the splitting of the product has to be done depending on a parameter $n \in \mathbb{N}$ in a somewhat tricky way, and estimates are required to be uniform with respect to $n$, cf. Remark 3.17.

The structure of the present paper is straightforward. First, in Section 2, we provide some preliminary facts about growth functions. In Section 3 we formulate and prove the main Theorem 3.3 and the supplements dealing with regularly distributed sequences. Finally, in Section 4 we present the aforementioned applications.

Acknowledgement. We thank Anton Baranov for discussion on the subject, and for communicating a stability result for well-separated sequences. The challenge to cover his result motivated us to push our method further until it reached the present form.

\section{Preliminaries about growth functions}

In complex analysis, in particular in the theory of entire functions, the notion of growth plays a central role.

2.1 Definition. A function $\lambda: \mathbb{R}^{+} \rightarrow \mathbb{R}^{+}$is called a growth function if it satisfies the following axioms:

(gf1) the limit $\rho_{\lambda}:=\lim _{r \rightarrow \infty} \frac{\log \lambda(r)}{\log r}$ exists and is finite and non-negative;

(gf2) for all sufficiently large values of $r$, the function $\lambda$ is differentiable and

$$
\lim _{r \rightarrow \infty}\left(r \frac{\lambda^{\prime}(r)}{\lambda(r)} / \frac{\log \lambda(r)}{\log r}\right)=1 ;
$$

moreover, $\lim _{r \rightarrow \infty} \lambda(r)=\infty$.

\footnotetext{
${ }^{4}$ Within Pontryagin space theory a variety of applications arises. In order not to overload the presentation, we decided not to touch upon these topics further.
} 
The conditions (gf1) and (gf2) ${ }^{5}$ ensure that we have Valiron's theory of proximate orders available, cf. [L1, Section I.12] or [LG, Section I.6]. Note that the logarithm of a growth function is often called proximate order.

Typical examples of growth functions are functions of the form

$$
\lambda(r)=r^{a} \cdot\left(\log _{\left(m_{1}\right)} r\right)^{b_{1}} \cdot \ldots \cdot\left(\log _{\left(m_{n}\right)} r\right)^{b_{n}}
$$

for large enough $r$; here $a \geq 0, m_{i} \in \mathbb{N}, m_{1}<\ldots<m_{n}, b_{1}, \ldots, b_{n} \in \mathbb{R}$, with $b_{1}>0$ if $a=0$, and $\log _{(n)}$ is defined by

$$
\log _{(1)} r:=\log r, \quad \log _{(k+1)} r:=\log \left(\log _{(k)} r\right), \quad k \in \mathbb{N},
$$

for large enough $r$. For the function in (2.1) we have $\rho_{\lambda}=a$.

As for the classical notion of order, also for general growth functions the growth of an entire function is related to the density of its zeros; see, e.g. $[\mathrm{Ru}$, Theorems 13.5.2-4]. Comparison of the growth of an entire function with functions of the form (2.1) goes back as far as to some work of E. Lindelöf in the early 20th century. However, in the present context we use growth functions only to handle the distribution of sequences.

2.2 Remark. Let $\lambda$ be a growth function. Then the following statements are true.

(i) $\lim _{r \rightarrow \infty} \frac{\lambda(C r)}{\lambda(r)}=C^{\rho_{\lambda}}$ uniformly in $C$ on compact subsets of $(0, \infty)$.

(ii) For sufficiently large values of $r$ the function $\lambda$ is strictly increasing.

(iii) Let $\sigma>0$; then, for sufficiently large $r$, the function $\frac{\lambda(r)}{r^{\sigma}}$ is increasing if $\sigma<\rho_{\lambda}$ and decreasing if $\sigma>\rho_{\lambda}$.

A proof of $(i)$ can be found in [L1, Lemma 5 in I.12] and [LG, Theorem 1.18, Proposition 1.19]. Item (ii) is a direct consequence of (gf2). Finally, (iii) follows from the relation

$$
\left[\frac{\lambda(r)}{r^{\sigma}}\right]^{\prime}=\frac{\lambda(r)}{r^{\sigma+1}}\left(-\sigma+\frac{r \lambda^{\prime}(r)}{\lambda(r)}\right)
$$

and the fact that $\lim _{r \rightarrow \infty} \frac{r \lambda^{\prime}(r)}{\lambda(r)}=\rho_{\lambda}$ by (gf2).

Property $(i)$ in Remark 2.2 says precisely that a growth function is a regularly varying function as defined, e.g. in [S, Definition 1.1]. However, we need the more special concept of a growth function since we use the properties (ii) and (iii) and the fact that $\lambda(r) \rightarrow \infty, r \rightarrow \infty$; regularly varying functions do not have the latter properties in general.

The whole importance of a growth function lies in its behaviour at $+\infty$. It is thus no loss of generality to assume additionally that

(gf3) the function $\lambda$ is differentiable, strictly increasing and bounded away from 0 .

\footnotetext{
${ }^{5}$ Instead of (gf2) often the condition $\lim _{r \rightarrow \infty} \frac{r \lambda^{\prime}(r)}{\lambda(r)}=\rho_{\lambda}$ is required. If $\rho_{\lambda}>0$, then, clearly, this is equivalent to (gf2). If $\rho_{\lambda}=0$, (gf2) is stronger.
} 
From now on we always include this property in the notion of a growth function. 2.3 Remark. In Subsection 2.2 we define sequences using the inverse of a growth function. Let us therefore state that a function $\mu:(a, \infty) \rightarrow(0, \infty)$ is the inverse of a growth function (satisfying (gf1)-(gf3)) if and only if

- $a>0 ; \mu$ is surjective and differentiable with positive derivative;

- $\rho_{\lambda}:=\lim _{s \rightarrow \infty} \frac{\log s}{\log \mu(s)}$ exists, is finite and non-negative;

- $\lim _{s \rightarrow \infty}\left(\frac{\mu(s)}{s \mu^{\prime}(s)} / \frac{\log s}{\log \mu(s)}\right)=1$.

\section{$2.1 \quad$ Upper and lower densities}

For a sequence $\xi$ of real numbers and a growth function $\lambda$, several densities are defined.

2.4 Definition. For a sequence $\xi=\left(\xi_{n}\right)_{n \in \mathbb{N}}$ of real numbers, set

$$
\begin{aligned}
& n_{\xi}^{+}(r):=\#\left\{n \in \mathbb{N}: 0<\xi_{n} \leq r\right\}, \\
& n_{\xi}^{-}(r):=\#\left\{n \in \mathbb{N}:-r \leq \xi_{n}<0\right\}, \\
& n_{\xi}(r):=\#\left\{n \in \mathbb{N}:\left|\xi_{n}\right| \leq r\right\} .
\end{aligned}
$$

The upper and lower right $\lambda$-densities of $\xi$ are defined as

$$
\Delta_{\lambda}^{+}(\xi):=\limsup _{r \rightarrow \infty} \frac{n_{\xi}^{+}(r)}{\lambda(r)}, \quad \delta_{\lambda}^{+}(\xi):=\liminf _{r \rightarrow \infty} \frac{n_{\xi}^{+}(r)}{\lambda(r)} .
$$

Similarly, the upper and lower left $\lambda$-densities are

$$
\Delta_{\lambda}^{-}(\xi):=\limsup _{r \rightarrow \infty} \frac{n_{\xi}^{-}(r)}{\lambda(r)}, \quad \delta_{\lambda}^{-}(\xi):=\liminf _{r \rightarrow \infty} \frac{n_{\xi}^{-}(r)}{\lambda(r)}
$$

and the upper and lower $\lambda$-densities are

$$
\Delta_{\lambda}(\xi):=\limsup _{r \rightarrow \infty} \frac{n_{\xi}(r)}{\lambda(r)}, \quad \delta_{\lambda}(\xi):=\liminf _{r \rightarrow \infty} \frac{n_{\xi}(r)}{\lambda(r)} .
$$

The following facts are elementary and are proved in the same way as [Bo, Lemma 1.5.1], which is nothing but the case when $\lambda(r)=r$. We skip the details.

2.5 Lemma. Let $\lambda$ be a growth function and let $\xi$ be a sequence of real numbers. Denote by $\xi^{+}$and $\xi^{-}$the (finite or infinite) subsequences of $\xi$ consisting of the positive or negative, respectively, elements of $\xi$ arranged according to increasing modulus and indexed with $n=1,2, \ldots$ Then ${ }^{6}$

$$
\begin{array}{rlrl}
\delta_{\lambda}^{+}(\xi) & =\liminf _{n \rightarrow \infty} \frac{n}{\lambda\left(\xi_{n}^{+}\right)}, & \Delta_{\lambda}^{+}(\xi)=\limsup _{n \rightarrow \infty} \frac{n}{\lambda\left(\xi_{n}^{+}\right)}, \\
\delta_{\lambda}^{-}(\xi)=\liminf _{n \rightarrow \infty} \frac{n}{\lambda\left(\left|\xi_{n}^{-}\right|\right)}, & \Delta_{\lambda}^{-}(\xi)=\limsup _{n \rightarrow \infty} \frac{n}{\lambda\left(\left|\xi_{n}^{-}\right|\right)} .
\end{array}
$$

${ }^{6}$ We tacitly understand the limit of a finite sequence as 0 . 
For a sequence $\xi$ we introduce two measures for the regularity of its distribution, the below defined values $s_{\xi}(n)$ and $r_{\xi}(\rho, n)$. The first is just the separation of the sequence, the second measures whether large lumps of points appear in $\xi$.

2.6 Definition. Let $\xi=\left(\xi_{n}\right)_{n \in \mathbb{N}}$ be a sequence of real non-zero numbers and let $\rho>1$. Then we define

$$
\begin{aligned}
s_{\xi}(n):=\inf \left\{\left|\xi_{k}-\xi_{n}\right|: k \in \mathbb{N}, \xi_{k} \neq \xi_{n}\right\}, & & n \in \mathbb{N}, \\
r_{\xi}(\rho, n):=\#\left\{k \in \mathbb{N}: \frac{\xi_{k}}{\xi_{n}} \in\left(\frac{1}{\rho}, \rho\right)\right\}, & & n \in \mathbb{N} .
\end{aligned}
$$

For each infinite sequence $\xi$ of real numbers that has no finite accumulation point, there exists a growth function $\lambda$ with $0<\Delta_{\lambda}(\xi)<\infty$, cf. [L1, Theorem 16 in I.12]. However, it need not be possible to choose $\lambda$ such that also $\delta_{\lambda}(\xi)>0$. This is related to the possible existence of large clusters of points in $\xi$. A quantitative statement is the following lemma.

2.7 Lemma. Let $\xi=\left(\xi_{n}\right)_{n \in \mathbb{N}}$ be an increasing sequence of positive real numbers that has no finite accumulation point. Let $\lambda$ be a growth function and assume that $\xi$ has finite upper and positive lower $\lambda$-densities. Then, for each $\rho>1$,

$$
r_{\xi}(\rho, n)=\mathrm{O}(n) \quad \text { and } \quad \sum_{\substack{k \in \mathbb{N} \\ \frac{\xi_{k}}{\xi_{n}} \in\left(\frac{1}{\rho}, \rho\right)}} \frac{1}{k}=\mathrm{O}(1), \quad n \rightarrow \infty .
$$

Proof. We set

$$
d_{1}:=\inf _{r \geq \xi_{1}} \frac{n_{\xi}(r)}{\lambda(r)}, \quad d_{2}:=\sup _{r \geq \xi_{1}} \frac{n_{\xi}(r)}{\lambda(r)}, \quad c_{\rho}:=\sup _{r \geq \xi_{1}} \frac{\lambda(\rho r)}{\lambda(r)} .
$$

Our assumption implies that $d_{1}>0$ and $d_{2}<\infty$, and Remark $2.2(i)$ and (gf3) imply that $1 \leq c_{\rho}<\infty$. Thus we can estimate

$$
r_{\xi}(\rho, n) \leq n_{\xi}\left(\rho \xi_{n}\right) \leq d_{2} \lambda\left(\rho \xi_{n}\right) \leq d_{2} c_{\rho} \lambda\left(\xi_{n}\right) \leq \frac{d_{2} c_{\rho}}{d_{1}} n_{\xi}\left(\xi_{n}\right)=\frac{d_{2} c_{\rho}}{d_{1}} \cdot n,
$$

which shows the first relation in (2.2). Next, set

$$
k_{+}(n):=\max \left\{k \in \mathbb{N}: \xi_{k} \leq \rho \xi_{n}\right\}, \quad k_{-}(n):=\min \left\{k \in \mathbb{N}: \frac{1}{\rho} \xi_{n}<\xi_{k}\right\} .
$$

Then

$$
k_{+}(n)=n_{\xi}\left(\rho \xi_{n}\right), \quad k_{-}(n)=n_{\xi}\left(\frac{1}{\rho} \xi_{n}\right)+1 .
$$

Moreover, let $n_{0} \in \mathbb{N}$ be such that $\frac{1}{\rho} \xi_{n_{0}} \geq \xi_{1}$. Then $k_{-}(n) \geq 2$ if $n \geq n_{0}$. For such $n$ we estimate

$$
\frac{k_{+}(n)}{k_{-}(n)-1}=\frac{n_{\xi}\left(\rho \xi_{n}\right)}{n_{\xi}\left(\frac{\xi_{n}}{\rho}\right)} \leq \frac{d_{2} \lambda\left(\rho \xi_{n}\right)}{d_{1} \lambda\left(\frac{\xi_{n}}{\rho}\right)} \leq \frac{d_{2} c_{\rho} \lambda\left(\xi_{n}\right)}{d_{1} \frac{1}{c_{\rho}} \lambda\left(\xi_{n}\right)}=\frac{d_{2} c_{\rho}^{2}}{d_{1}}
$$


Clearly, $\lim _{n \rightarrow \infty} k_{+}(n)=\lim _{n \rightarrow \infty} k_{-}(n)=\infty$, and hence (here $\gamma$ denotes the Euler-Mascheroni constant)

$$
\begin{aligned}
\sum_{k \in \mathbb{N}: \frac{1}{\rho} \xi_{n}<\xi_{k} \leq \rho \xi_{n}} \frac{1}{k} & =\sum_{k=k_{-}(n)}^{k_{+}(n)} \frac{1}{k} \\
& =\left[\log k_{+}(n)+\gamma+\mathrm{o}(1)\right]-\left[\log \left(k_{-}(n)-1\right)+\gamma+\mathrm{o}(1)\right] \\
& =\log \frac{k_{+}(n)}{k_{-}(n)-1}+\mathrm{o}(1)=\mathrm{O}(1)
\end{aligned}
$$

as $n \rightarrow \infty$.

2.8 Definition. We call a sequence $\xi$ of convergence class with respect to a growth function $\lambda$ if

$$
\sum_{n \in \mathbb{N}} \frac{1}{\lambda\left(\left|\xi_{n}\right|\right)}<\infty .
$$

For example, the sequence $\xi_{n}:=n^{\frac{1}{\rho}}$ where $\rho>0$ is of convergence class with respect to the growth function $\lambda$ that satisfies $\lambda(r):=r^{\rho} \log r(\log \log r)^{2}$ for large $r$, but it is not of convergence class with respect to $\lambda(r):=r^{\rho}$.

The next statement is an analogue of the classical case when $\lambda(r)=r^{\rho}$ and is proved in the same way, cf. [Bo, Proof of Lemma 2.5.5]. We again skip the details.

2.9 Lemma. Assume that $\xi$ is of convergence class with respect to the growth function $\lambda$. Then

$$
n_{\xi}(r)=\mathrm{O}(\lambda(r)), \quad r \rightarrow \infty .
$$

The converse of this fact is not true (as already seen from the above mentioned example).

\subsection{The standard sequence $\lambda$}

With a growth function we associate a sequence that has most regular behaviour with respect to $\lambda$.

2.10 Definition. Let $\lambda$ be a growth function. Then we define the standard sequence $\stackrel{\AA}{\lambda}=\left(\AA_{n}\right)_{n \in \mathbb{N}}$ associated with $\lambda$ by

$$
\grave{\lambda}_{n}:=\lambda^{-1}(n), \quad n \in \mathbb{N} .
$$

First, we collect some simple properties of this sequence. Recall that the convergence exponent of a sequence $\xi$ of non-zero numbers is defined as

$$
\inf \left\{\rho>0: \sum_{n=1}^{\infty} \frac{1}{\left|\xi_{n}\right|^{\rho}}<\infty\right\} .
$$


2.11 Lemma. Let $\lambda$ be a growth function, and let $\dot{\lambda}$ be the standard sequence associated with $\lambda$. Moreover, let $\rho_{\lambda}$ be as in (gf1). Then the following statements hold.

(i) The sequence $\stackrel{\lambda}{\lambda}$ is strictly increasing.

(ii) We have $n_{\dot{\lambda}}(r)=\lfloor\lambda(r)\rfloor$; here $\lfloor x\rfloor$ denotes the largest integer less than or equal to $x$.

(iii) We have $\Delta_{\lambda}(\stackrel{\circ}{\lambda})=\delta_{\lambda}(\stackrel{\circ}{\lambda})=1$.

(iv) The convergence exponent of $\dot{\lambda}$ is equal to $\rho_{\lambda}$.

Proof. Item $(i)$ is clear from our additional axiom (gf3). For item $(i i)$, let $n \in \mathbb{N}$. Since $\grave{\lambda}_{n}=\lambda^{-1}(n)$, we have $\dot{\lambda}_{n} \leq r$ if and only if $n \leq \lambda(r)$. This shows that $n_{\grave{\lambda}}(r)=\lfloor\lambda(r)\rfloor$. Item $(i i i)$ is obvious from $(i i)$, and item $(i v)$ follows since the convergence exponent of $\stackrel{\lambda}{\lambda}$ can be computed as $\rho_{1}=\lim \sup _{r \rightarrow \infty} \frac{\log n_{\hat{\lambda}}(r)}{\log r}$, see, e.g. [Bo, Theorem 2.5.8].

For standard sequences we can control $s_{\grave{\lambda}}$ and $r_{\grave{\lambda}}$. These estimates are used in the proof of Theorem 3.19.

2.12 Lemma. Let $\lambda$ be a growth function, and let $\rho>1$. Then

$$
\frac{1}{s_{\grave{\lambda}}(n)}=\mathrm{O}\left(\frac{n}{\dot{\lambda}_{n}}\right), \quad \sum_{\substack{k \in \mathbb{N}: k \neq n \\ \frac{\lambda_{k}}{\lambda_{n}} \in\left(\frac{1}{\rho}, \rho\right)}} \frac{1}{\left|\stackrel{\lambda}{\lambda}_{k}-\stackrel{\circ}{\lambda}_{n}\right|}=\mathrm{O}\left(\frac{n \log n}{\stackrel{\circ}{\lambda}_{n}}\right), \quad n \rightarrow \infty .
$$

Proof. Let $k, n \in \mathbb{N}$. We use the mean value theorem to obtain a point $\theta_{k, n}$ between $k$ and $n$ with

$$
\stackrel{\circ}{\lambda}_{k}-\grave{\lambda}_{n}=\lambda^{-1}(k)-\lambda^{-1}(n)=\frac{k-n}{\lambda^{\prime}\left(\lambda^{-1}\left(\theta_{k, n}\right)\right)} .
$$

Due to (gf2) we have $\lambda^{\prime}(r) \leq C \frac{\lambda(r)}{r}$ with some $C>0$, and hence

$$
\lambda^{\prime}\left(\lambda^{-1}\left(\theta_{k, n}\right)\right) \leq C \frac{\theta_{k, n}}{\lambda^{-1}\left(\theta_{k, n}\right)} .
$$

For $k=n+1$ this yields

$$
\begin{aligned}
\frac{1}{\left|\stackrel{\circ}{\lambda+1}_{n+1}-\stackrel{\circ}{n}_{n}\right|} & =\lambda^{\prime}\left(\lambda^{-1}\left(\theta_{n+1, n}\right)\right) \leq C \frac{\theta_{n+1, n}}{\lambda^{-1}\left(\theta_{n+1, n}\right)} \\
& \leq C \frac{n+1}{\lambda^{-1}(n)}=C \frac{n+1}{\stackrel{\circ}{\lambda}_{n}} .
\end{aligned}
$$

If $\stackrel{\lambda}{n-1}_{n} \geq \frac{1}{\rho} \stackrel{\lambda}{n}_{n}$, then (2.6) and (2.7) with $k=n-1$ yield

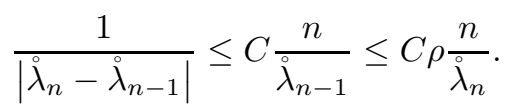


If $\grave{\lambda}_{n-1}<\frac{1}{\rho} \grave{\lambda}_{n}$, then

$$
\frac{1}{\left|\grave{\lambda}_{n}-\grave{\lambda}_{n-1}\right|}=\frac{1}{\left(1-\frac{\check{\lambda}_{n-1}}{\dot{\lambda}_{n}}\right) \grave{\lambda}_{n}} \leq \frac{1}{\left(1-\frac{1}{\rho}\right) \grave{\lambda}_{n}} .
$$

This together with (2.8) and (2.9) shows the first relation in (2.5).

Due to Lemma 2.11 (iii) we can apply Lemma 2.7 and obtain $r_{\lambda}(\rho, n)=$ $\mathrm{O}(n)$. Assume that $\frac{\stackrel{\iota}{\lambda}_{k}}{\dot{\lambda}_{n}} \in\left(\frac{1}{\rho}, \rho\right)$. With $c_{\rho}$ as in (2.3) we have

$$
\frac{k}{n}=\frac{\lambda\left(\stackrel{\circ}{\lambda}_{k}\right)}{\lambda\left(\grave{\lambda}_{n}\right)} \leq \frac{\lambda\left(\rho \stackrel{\lambda}{\lambda}_{n}\right)}{\lambda\left(\stackrel{\circ}{n}_{n}\right)} \leq c_{\rho},
$$

which implies that $\theta_{k, n} \leq c_{\rho} n$. Moreover, $\lambda^{-1}\left(\theta_{k, n}\right)$ is some point between $\grave{\lambda}_{k}$ and $\stackrel{\circ}{\lambda}_{n}$, and hence $\lambda^{-1}\left(\theta_{k, n}\right) \geq \frac{1}{\rho} \AA_{n}$. Hence

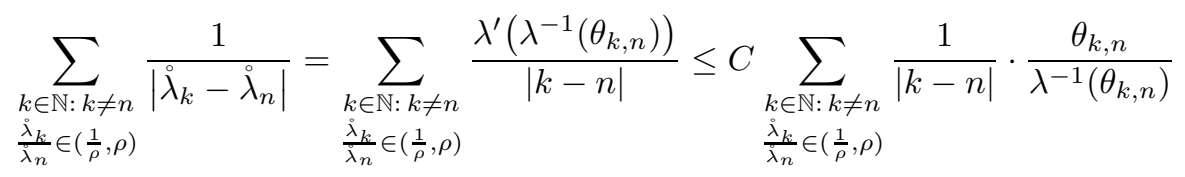

$$
\begin{aligned}
& \leq C \frac{c_{\rho} n}{\frac{1}{\rho} \grave{\lambda}_{n}} \sum_{\substack{k \in \mathbb{N}: k \neq n \\
\frac{\lambda_{k}}{\lambda_{n}} \in\left(\frac{1}{\rho}, \rho\right)}} \frac{1}{|k-n|} \leq C \frac{\rho c_{\rho} n}{\grave{\lambda}_{n}} \cdot 2 \sum_{l=1}^{r_{\grave{\lambda}}(\rho, n)} \frac{1}{l} \lesssim \frac{n \log n}{\grave{\lambda}_{n}}
\end{aligned}
$$

as $n \rightarrow \infty$.

Next, let us investigate growth functions $\Lambda$ such that $\lambda$ is of convergence class with respect to $\Lambda$. Since

$$
\sum_{n \in \mathbb{N}} \frac{1}{\lambda\left(\stackrel{\circ}{n}_{n}\right)}=\sum_{n \in \mathbb{N}} \frac{1}{n}=\infty
$$

one has to choose a slightly larger growth function in order to achieve convergence class. For example, the choices $\Lambda(r)=\lambda(r)(\log \lambda(r))^{2}$ and $\Lambda(r)=$ $\lambda(r) \log \lambda(r)(\log \log \lambda(r))^{2}$, for large $r$, will always do the job.

The next statement can be seen as a refinement of Lemma 2.9. Thinking of the example below Definition 2.8, this fact is no surprise. This estimate is used in Corollary 3.20 below.

2.13 Lemma. Let $\lambda$ and $\Lambda$ be growth functions such that the standard sequence $\lambda$ is of convergence class with respect to $\Lambda$. Assume that $\frac{\lambda}{\Lambda}$ is, for sufficiently large values of $r$, non-increasing.

If $\rho_{\lambda}>0$ or the function $\frac{\log \lambda(r)}{\log r}$ is non-increasing for large $r$, then

$$
\lambda(r) \log \lambda(r)=\mathrm{O}(\Lambda(r)), \quad r \rightarrow \infty .
$$

Proof. Let $n \in \mathbb{N}$. Then

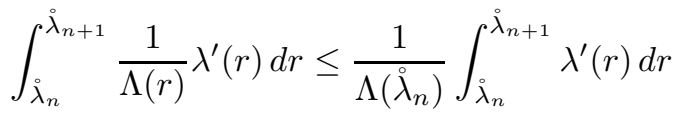

$$
\begin{aligned}
& =\frac{1}{\Lambda\left(\stackrel{\circ}{\lambda}_{n}\right)}\left(\lambda\left(\stackrel{\circ}{\lambda}_{n+1}\right)-\lambda\left(\stackrel{\circ}{\lambda}_{n}\right)\right)=\frac{1}{\Lambda\left(\stackrel{\circ}{\lambda}_{n}\right)},
\end{aligned}
$$


which implies that $\int_{1}^{\infty} \frac{1}{\Lambda(r)} \lambda^{\prime}(r) d r<\infty$.

First, assume that $\rho_{\lambda}>0$. Then we find $r_{0}>1$ such that $\frac{\lambda(r)}{\Lambda(r)}$ is nonincreasing for $r \geq r_{0}$ and, by (gf2),

$$
\lambda^{\prime}(r) \geq \frac{\rho_{\lambda}}{2} \frac{\lambda(r)}{r}, \quad r \geq r_{0} .
$$

Hence, $\int_{r_{0}}^{\infty} \frac{1}{\Lambda(r)} \frac{\lambda(r)}{r} d r<\infty$, and integrating by parts gives $\left(R \geq r_{0}\right)$

$$
\begin{aligned}
\infty & >\int_{r_{0}}^{\infty} \frac{1}{\Lambda(r)} \frac{\lambda(r)}{r} d r \geq \int_{r_{0}}^{R} \frac{\lambda(r)}{\Lambda(r)} \cdot \frac{1}{r} d r \\
& =\frac{\lambda(R)}{\Lambda(R)} \log R-\frac{\lambda\left(r_{0}\right)}{\Lambda\left(r_{0}\right)} \log r_{0}-\int_{r_{0}}^{R} \underbrace{\left(\frac{\lambda(r)}{\Lambda(r)}\right)^{\prime}}_{\leq 0} \log r d r .
\end{aligned}
$$

We see that $\frac{\lambda(R)}{\Lambda(R)} \log R$ remains bounded when $R$ tends to $\infty$, and hence (2.10) holds since $\lambda$ satisfies (gf1).

Assume now that $\frac{\log \lambda(r)}{\log r}$ is non-increasing (for large $r$ ). Then we can choose $r_{0}>1$ such that $\frac{\lambda(r)}{\Lambda(r)}$ and $\frac{\log \lambda(r)}{\log r}$ are both non-increasing for $r \geq r_{0}$ and that

$$
\lambda^{\prime}(r) \geq \frac{1}{2} \frac{\lambda(r) \log \lambda(r)}{r \log r}, \quad r \geq r_{0} .
$$

Again we integrate by parts and obtain

$$
\begin{aligned}
\infty & >2 \int_{r_{0}}^{\infty} \frac{1}{\Lambda(r)} \lambda^{\prime}(r) d r \geq \int_{r_{0}}^{R} \frac{\lambda(r)}{\Lambda(r)} \frac{\log \lambda(r)}{\log r} \cdot \frac{1}{r} d r \\
& =\frac{\lambda(R) \log \lambda(R)}{\Lambda(R)}-\frac{\lambda\left(r_{0}\right) \log \lambda\left(r_{0}\right)}{\lambda\left(r_{0}\right)}-\int_{r_{0}}^{R} \underbrace{\left(\frac{\lambda(r)}{\Lambda(r)} \frac{\log \lambda(r)}{\log r}\right)^{\prime}}_{\leq 0} \cdot \log r d r,
\end{aligned}
$$

which, also in this case, shows that $\frac{\lambda(R) \log \lambda(R)}{\Lambda(R)}$ is bounded.

2.14 Remark. The hypothesis of this Lemma 2.13 is satisfied in 'most' cases. (1) In order to construct a growth function $\Lambda$ which makes $\lambda$ of convergence class, it is natural to multiply $\lambda$ with some growing factor, in which case $\frac{\lambda(r)}{\Lambda(r)}$ is non-increasing. (2) Assume that $\rho_{\lambda}=0$. Then $\frac{\log \lambda(r)}{\log r}$ is a positive function (for large $r$ ), which tends to zero. Thinking of $\lambda$ as a regularly behaving function, requiring monotonicity appears to be not too restrictive.

If $\lambda$ is given by its inverse function $\mu$, then one can characterize the assumptions in Lemma 2.13 in terms of $\mu$. Namely, $\frac{\lambda(r)}{\Lambda(r)}$ is non-increasing if and only if $\frac{\Lambda(\mu(s))}{s}$ is non-decreasing; $\frac{\log \lambda(r)}{\log r}$ is non-increasing if and only if $\frac{\log \mu(s)}{\log s}$ is non-decreasing. Note also that if $\mu(s)=\mathrm{O}\left(s^{\sigma}\right), s \rightarrow \infty$, for some $\sigma>0$, then $\rho_{\lambda} \geq \frac{1}{\sigma}>0$. 


\section{Stability theorems}

Let us introduce the precise setup.

3.1 Definition. We denote by $\mathbb{S}$ the set of all sequences $\xi=\left(\xi_{n}\right)_{n=1}^{\infty}$ of real numbers that satisfy the following conditions.

(S1) The sequence $\xi$ consists of pairwise distinct non-zero points and has no finite accumulation point.

(S2) Denote by $\xi^{+}$and $\xi^{-}$the (finite or infinite) subsequences of $\xi$ consisting of all positive or negative, respectively, elements of $\xi$ arranged according to increasing modulus ${ }^{7}$. Then

$$
\lim _{n \rightarrow \infty} \frac{n}{\xi_{n}^{+}}=\lim _{n \rightarrow \infty} \frac{n}{\left|\xi_{n}^{-}\right|} \in[0, \infty),
$$

where we tacitly understand the limit of a finite sequence as being equal to 0 .

(S3) The limit

$$
\lim _{r \rightarrow \infty} \sum_{\left|\xi_{n}\right| \leq r} \frac{1}{\xi_{n}}
$$

exists in $\mathbb{R}$.

Note that we assume no particular ordering of $\xi$; only $\xi^{+}$and $\xi^{-}$are ordered with increasing modulus. We also mention that (S2) is equivalent to

$$
\delta_{\lambda}^{+}(\xi)=\delta_{\lambda}^{-}(\xi)=\Delta_{\lambda}^{+}(\xi)=\Delta_{\lambda}^{-}(\xi) \in[0, \infty)
$$

for $\lambda(r)=r$.

With each sequence $\xi \in \mathbb{S}$ we associate an entire function $P_{\xi}$, namely the canonical product connected with $\xi$.

3.2 Definition. Let $\xi \in \mathbb{S}$. Then we set

$$
P_{\xi}(z):=\lim _{r \rightarrow \infty} \prod_{\left|\xi_{n}\right| \leq r}\left(1-\frac{z}{\xi_{n}}\right), \quad z \in \mathbb{C} .
$$

Because of (S1)-(S3), this limit exists locally uniformly on $\mathbb{C}$ and represents an entire function of finite exponential type whose zeros are all simple and located exactly at the points $\xi_{n}, n \in \mathbb{N}$.

The following statement is the main result of this paper.

3.3 Theorem. Let $\alpha \in \mathbb{S}$ and let $\Lambda$ be a growth function such that, for sufficiently large $r$, the function $\frac{\Lambda(r)}{r}$ is either non-increasing or non-decreasing. Moreover, assume that $\alpha$ is of convergence class with respect to $\Lambda$, i.e.

$$
\sum_{n \in \mathbb{N}} \frac{1}{\Lambda\left(\left|\alpha_{n}\right|\right)}<\infty
$$

\footnotetext{
${ }^{7}$ Both sequences are supposed to have finite or infinite index sets of the form $1,2, \ldots$.
} 
Further, let $\beta \in \mathbb{S}$ be a small perturbation of $\alpha$ in the sense that the difference $\gamma:=\beta-\alpha$ satisfies the following conditions:

$$
\begin{aligned}
& \left|\gamma_{n}\right|=\mathrm{O}\left(\frac{\left|\alpha_{n}\right|}{\Lambda\left(\left|\alpha_{n}\right|\right)}\right), \quad n \rightarrow \infty ; \\
& \left(\frac{\gamma_{n}}{s_{\alpha}(n)}\right)_{n \in \mathbb{N}} \in \ell^{1}, \\
& \exists \rho>1: \frac{\gamma_{n}}{s_{\alpha}(n)}=\mathrm{O}\left(\frac{1}{r_{\alpha}(\rho, n)}\right), \quad n \rightarrow \infty,
\end{aligned}
$$

where $s_{\alpha}$ and $r_{\alpha}$ are defined as in Definition 2.6. Let $P_{\alpha}$ and $P_{\beta}$ be the canonical products associated with $\alpha$ and $\beta$, respectively. Then

$$
\left|P_{\alpha}^{\prime}\left(\alpha_{n}\right)\right| \asymp\left|P_{\beta}^{\prime}\left(\beta_{n}\right)\right|, \quad n \rightarrow \infty .
$$

3.4 Remark.

(i) If $\rho_{\Lambda}<1$, then $\frac{\Lambda(r)}{r}$ is decreasing for large $r$ by Remark $2.2($ iii $)$. In this case, Condition $(\mathrm{A})$ allows $\gamma$ to grow (although the conditions in (B) still have to be satisfied, which may prohibit this). If $\frac{\Lambda(r)}{r}$ is non-decreasing, then $\gamma$ must be a bounded sequence.

(ii) Let us comment on the role of the condition (S2) and (S3) which are required from the start. What we want to do (and what we are able to do) is to estimate the derivative of functions which are of finite exponential type and represented as a product $P_{\xi}$, and not of canonical products which include exponential factors. In order to ensure that $P_{\xi}$ convergences and is of finite exponential type, of course some requirements on $\xi$ are necessary, namely at least that the genus of the sequence $\xi$ does not exceed 1 and that (S3) holds. Condition (S2) on existence and equality of angular densities is of course not necessary purely for convergence. It is related to regular growth: in the applications which motivated us to study this stability question at all, sequences are zero sequences of functions of Cartwright class and thus satisfy (S2).

We should point out that, as soon as we deal with (zero sequences of) functions of minimal exponential type, both conditions (S2) and (S3) are automatic (by Lindelöf's theorem).

3.5 Remark. The following intuitive picture can be regarded as commonly accepted (believed):

At places in the vicinity of which the sequence $\alpha$ is well separated the associated sequence $\alpha^{\prime}=\left(P_{\alpha}^{\prime}\left(\alpha_{n}\right)\right)_{n \in \mathbb{N}}$ behaves regularly and can be controlled. On the other hand, points of $\alpha$ being close to each other give rise to peaks in $\alpha^{\prime}$, and lumps of points being close to each other produce peaks which even may spread out over neighbouring points.

Hence, it is to be expected that a perturbation of $\alpha$ which does not influence the behaviour of $\alpha^{\prime}$ should be asymptotically smaller than the separation $s_{\alpha}$ of $\alpha$. The perturbation must certainly be limited by the separation of $\alpha$ 
because otherwise, we could remove or add zeros, which definitely changes the asymptotic behaviour of $\alpha^{\prime}$.

The conditions (A) and (B) are quantitative instantiations of this idea. For (B) this is obvious: (3.3) rules out perturbations which produce close points, and (3.4) rules out that lumps of points appear in the vicinity of $\alpha_{n}$. To understand (A), it is advisable to consider an example of a very regularly distributed sequence where all involved quantities can be computed explicitly. Take, e.g. the sequence $\alpha_{n}:=n^{\sigma}$ with some $\sigma>1$. Then $s_{\alpha}(n) \asymp n^{\sigma-1}$, and we may choose for $\Lambda$, e.g. $\Lambda(r):=r^{\frac{1}{\sigma}} \log r(\log \log r)^{2}$. Condition (3.2) becomes

$$
\frac{\left|\gamma_{n}\right|}{s_{\alpha}(n)}=\mathrm{O}\left(\frac{1}{\log n(\log \log n)^{2}}\right) \text {. }
$$

We see that (A) requires the perturbation to be only slightly smaller than the separation, but, contrasting (3.3), that $\frac{\left|\gamma_{n}\right|}{s_{\alpha}(n)}$ tends to zero in a controllably regular way. We discuss more examples in Subsection 3.3.

We split the proof of Theorem 3.3 in two subsections.

\subsection{The basic estimates}

First note that

$$
P_{\xi}^{\prime}\left(\xi_{n}\right)=-\frac{1}{\xi_{n}} \lim _{r \rightarrow \infty} \prod_{\substack{\left|\xi_{k}\right| \leq r \\ k \neq n}}\left(1-\frac{\xi_{n}}{\xi_{k}}\right), \quad n \in \mathbb{N} .
$$

In this subsection we provide two general estimates for quotients of such products; see Propositions 3.6 and 3.9 below. The first one deals with factors where $\xi_{k}$ is small.

3.6 Proposition. Let the following data be given:

- a sequence $\xi=\left(\xi_{n}\right)_{n \in \mathbb{N}}$ of non-zero real numbers which has no finite accumulation point;

- a growth function $\Lambda$ such that $\xi$ is of convergence class with respect to $\Lambda$;

- a sequence $\nu=\left(\nu_{n}\right)_{n \in \mathbb{N}}$ of real numbers with $\nu_{n} \neq-\xi_{n}, n \in \mathbb{N}$.

Denote by $r_{n}$ the unique positive numbers with $r_{n} \Lambda\left(r_{n}\right)=\left|\xi_{n}\right|$, let $c>0$ and set

$$
J_{n}(c, \Lambda):=\left\{k \in \mathbb{N}:\left|\xi_{k}\right| \leq c r_{n}\right\} .
$$

Moreover, set $\eta_{n}:=\xi_{n}+\nu_{n}, n \in \mathbb{N}$.

If the sequence $\nu$ is subject to the condition

$$
\left|\nu_{n}\right|=\mathrm{O}\left(\frac{\left|\xi_{n}\right|}{\Lambda\left(\left|\xi_{n}\right|\right)}\right), \quad n \rightarrow \infty
$$

then

$$
0<\liminf _{n \rightarrow \infty}\left|\prod_{k \in J_{n}(c, \Lambda)} \frac{1-\frac{\eta_{n}}{\eta_{k}}}{1-\frac{\xi_{n}}{\xi_{k}}}\right| \leq \limsup _{n \rightarrow \infty}\left|\prod_{k \in J_{n}(c, \Lambda)} \frac{1-\frac{\eta_{n}}{\eta_{k}}}{1-\frac{\xi_{n}}{\xi_{k}}}\right|<\infty .
$$


It is shown in the proof below that $n \notin J_{n}(c, \Lambda)$ if $n$ is large enough, so that the products are well defined for such $n$.

We frequently use the standard procedure to estimate products by taking logarithms. The following remark is of course trivial; however, since our estimates have to be uniform with respect to several parameters, it is better to be precise (we return to this note in Remark 3.17).

3.7 Remark. Let $I$ be a finite subset of $\mathbb{N}$, and let $x_{n}, n \in I$, be real numbers with $\left|x_{n}\right| \leq \frac{1}{2}$. Then

$$
|\log | \prod_{n \in I}\left(1+x_{n}\right)|| \leq \log 4 \cdot \sum_{n \in I}\left|x_{n}\right| .
$$

This is obvious from the fact that

$$
\log |1+x| \begin{cases}\leq \log (1+|x|) \leq|x|, & x \in \mathbb{R}, \\ \geq \log (1-|x|) \geq-(\log 4)|x|, & |x| \leq \frac{1}{2} .\end{cases}
$$

Of course, the inequality in (3.9) then leads to the estimate

$$
e^{-\log 4 \sum_{n \in I}\left|x_{n}\right|} \leq\left|\prod_{n \in I}\left(1+x_{n}\right)\right| \leq e^{\log 4 \sum_{n \in I}\left|x_{n}\right|} .
$$

Proof of Proposition 3.6. First, we collect some simple facts.

(i) The numbers $r_{n}$ are indeed well defined since the function $r \Lambda(r)$ is a bijection from $(0, \infty)$ onto itself. Moreover, $\lim _{n \rightarrow \infty} r_{n}=\infty$.

(ii) We have $\frac{r_{n}}{\left|\xi_{n}\right|}=\frac{1}{\Lambda\left(r_{n}\right)} \rightarrow 0$. In particular, for $n$ sufficiently large, $r_{n} \leq\left|\xi_{n}\right|$. For such values of $n$,

$$
\frac{r_{n}}{\left|\xi_{n}\right|}=\frac{1}{\Lambda\left(r_{n}\right)} \geq \frac{1}{\Lambda\left(\left|\xi_{n}\right|\right)}
$$

and hence $r_{n} \geq \frac{\left|\xi_{n}\right|}{\Lambda\left(\left|\xi_{n}\right|\right)}$.

(iii) We have $\frac{\left|\nu_{n}\right|}{\left|\xi_{n}\right|}=\mathrm{O}\left(\frac{1}{\Lambda\left(\xi_{n}\right)}\right)$, and hence

$$
\lim _{n \rightarrow \infty} \frac{\left|\nu_{n}\right|}{\left|\xi_{n}\right|}=0 \quad \text { and } \quad \lim _{n \rightarrow \infty} \frac{\left|\eta_{n}\right|}{\left|\xi_{n}\right|}=1
$$

(iv) Let $c>0$. Then, by (ii), there exists an $n_{0} \in \mathbb{N}$ such that $r_{n} \leq \frac{\xi_{n}}{4 c}$, $n \geq n_{0}$, and hence

$$
\left|\xi_{k}\right| \leq \frac{1}{4}\left|\xi_{n}\right|, \quad k \in J_{n}(c, \Lambda), n \geq n_{0} .
$$

Using (3.11) we conclude that there exists an $n_{1} \in \mathbb{N}$ such that

$$
\left|\eta_{k}\right| \leq \frac{1}{2}\left|\eta_{n}\right|, \quad k \in J_{n}(c, \Lambda), n \geq n_{1} .
$$


(v) For $n \geq n_{0}$ we have $k \notin J_{n}(c, \Lambda)$ whenever $\left|\xi_{k}\right|=\left|\xi_{n}\right|$. In particular, $n \notin J_{n}(c, \Lambda)$. For such indices $n$, the products in (3.8) are well defined.

(vi) For each finite subset $M \subseteq \mathbb{N}$, there exists an $n_{2} \in \mathbb{N}$ such that

$$
M \subseteq J_{n}(c, \Lambda), \quad n \geq n_{2} .
$$

(vii) By Lemma 2.9 we have

$$
\# J_{n}(c, \Lambda)=n_{\xi}\left(c r_{n}\right)=\mathrm{O}\left(\Lambda\left(r_{n}\right)\right), \quad n \rightarrow \infty .
$$

Now we come to the actual proof of Proposition 3.6. Choose $N \in \mathbb{N}$ such that

$$
\begin{aligned}
& \frac{\left|\nu_{n}\right|}{\left|\xi_{n}\right|} \leq\left[4 \sup _{l \in \mathbb{N}} \frac{\left|\xi_{l}\right|}{\left|\eta_{l}\right|}\right]^{-1}, \quad \frac{\left|\nu_{n}\right|}{\left|\eta_{n}\right|} \leq \frac{1}{4}, \quad n \geq N, \\
& \frac{\left|\xi_{k}\right|}{\left|\xi_{n}\right|} \leq \frac{1}{2}, \quad \frac{\left|\eta_{k}\right|}{\left|\eta_{n}\right|} \leq \frac{1}{2}, \quad n \geq N, k \in J_{n}(c, \Lambda),
\end{aligned}
$$

and choose $N_{1} \in \mathbb{N}, N_{1} \geq N$, such that

$$
\{k \in \mathbb{N}: k<N\} \subseteq J_{n}(c, \Lambda), \quad n \geq N_{1} .
$$

We rewrite (using the shorthand $J_{n}:=J_{n}(c, \Lambda)$ )

$$
\prod_{k \in J_{n}} \frac{1-\frac{\eta_{n}}{\eta_{k}}}{1-\frac{\xi_{n}}{\xi_{k}}}=\frac{\prod_{k \in J_{n}}\left(\frac{\eta_{k}}{\eta_{n}}-1\right)}{\prod_{k \in J_{n}}\left(\frac{\xi_{k}}{\xi_{n}}-1\right)} \cdot \prod_{\substack{k \in J_{n} \\ k \geq N}} \frac{\xi_{k} \eta_{n}}{\xi_{n} \eta_{k}} \cdot \prod_{k<N} \frac{\xi_{k} \eta_{n}}{\xi_{n} \eta_{k}}, \quad n \geq N_{1}
$$

and consider each factor separately.

For the product in the denominator of the first factor, we estimate

$$
\sum_{k \in J_{n}}\left|\frac{\xi_{k}}{\xi_{n}}\right| \leq \frac{\max _{k \in J_{n}}\left|\xi_{k}\right|}{\left|\xi_{n}\right|} \cdot\left(\# J_{n}\right) \lesssim \frac{c r_{n}}{\left|\xi_{n}\right|} \cdot \Lambda\left(r_{n}\right)=c .
$$

For the product in the numerator, note that

$$
\left|\frac{\eta_{k}}{\eta_{n}}\right| \leq \sup _{k \in \mathbb{N}}\left|\frac{\eta_{k}}{\xi_{k}}\right| \cdot \sup _{n \in \mathbb{N}}\left|\frac{\xi_{n}}{\eta_{n}}\right| \cdot\left|\frac{\xi_{k}}{\xi_{n}}\right| \cdot
$$

Hence, the same estimate applies.

Next, we write

$$
\frac{\xi_{k} \eta_{n}}{\xi_{n} \eta_{k}}=1+\frac{\xi_{k}\left(\eta_{n}-\xi_{n}\right)-\left(\eta_{k}-\xi_{k}\right) \xi_{n}}{\xi_{n} \eta_{k}}=1+\frac{\xi_{k}}{\eta_{k}} \frac{\nu_{n}}{\xi_{n}}-\frac{\nu_{k}}{\eta_{k}},
$$

and, using (ii), (iii) and (vii), we can estimate

$$
\begin{aligned}
& \sum_{k \in J_{n}}\left|\frac{\xi_{k}}{\eta_{k}} \frac{\nu_{n}}{\xi_{n}}\right| \leq \frac{\left|\nu_{n}\right|}{\left|\xi_{n}\right|} \cdot\left(\# J_{n}\right) \sup _{k \in \mathbb{N}}\left|\frac{\xi_{k}}{\eta_{k}}\right| \cdot \lesssim \frac{\left|\nu_{n}\right|}{\left|\xi_{n}\right|} \Lambda\left(\left|r_{n}\right|\right) \lesssim \frac{\left|\nu_{n}\right|}{\left|\xi_{n}\right|} \Lambda\left(\left|\xi_{n}\right|\right)=\mathrm{O}(1), \\
& \sum_{k \in J_{n}}\left|\frac{\nu_{k}}{\eta_{k}}\right| \leq \sup _{k \in \mathbb{N}}\left|\frac{\xi_{k}}{\eta_{k}}\right| \cdot \sum_{k \in J_{n}}\left|\frac{\nu_{k}}{\xi_{k}}\right| \lesssim \sum_{k \in J_{n}} \frac{1}{\Lambda\left(\left|\xi_{k}\right|\right)} \leq \sum_{k \in \mathbb{N}} \frac{1}{\Lambda\left(\left|\xi_{k}\right|\right)}<\infty .
\end{aligned}
$$


By our choice of $N$, we have, for $n \geq N, k \in J_{n}, k \geq N$, that

$$
\left|\frac{\xi_{k}}{\eta_{k}} \frac{\nu_{n}}{\xi_{n}}-\frac{\nu_{k}}{\eta_{k}}\right| \leq \frac{\left|\nu_{n}\right|}{\left|\xi_{n}\right|} \sup _{l \in \mathbb{N}} \frac{\left|\xi_{l}\right|}{\left|\eta_{l}\right|}+\sup _{l \geq N} \frac{\left|\nu_{l}\right|}{\left|\eta_{l}\right|} \leq \frac{1}{2}
$$

and hence Remark 3.7 can be applied to the second factor in (3.12). The same is true for the products in the first factor. Hence the estimate (3.10) implies that the products in the first three factors are bounded from above and away from zero uniformly in $n$.

It remains to notice that

$$
\lim _{n \rightarrow \infty} \prod_{k<N} \frac{\xi_{k} \eta_{n}}{\xi_{n} \eta_{k}}=\prod_{k<N} \frac{\xi_{k}}{\eta_{k}} \neq 0 .
$$

Proposition 3.9 below contains a key estimate. In its proof we use the following fact.

3.8 Lemma. Let $\Lambda$ be a growth function, $\rho>1$ and $R>0$ such that $\frac{\Lambda(r)}{r}$ is non-decreasing and $\frac{\Lambda(r)}{r^{2}}$ is non-increasing on $[R, \infty)$. Then

$$
\left|\frac{b}{a(a-b)}\right| \leq \frac{\rho}{\rho-1} \cdot \frac{\Lambda(|b|)}{|b| \Lambda(|a|)}, \quad a, b \in \mathbb{R}, \quad|a|,|b| \geq R, \quad \frac{a}{b} \notin\left(\frac{1}{\rho}, \rho\right) .
$$

Proof. We first consider the case when $a, b>0$. If $\frac{a}{b} \leq \frac{1}{\rho}$, then $|a-b|=$ $\left(1-\frac{a}{b}\right) b \geq\left(1-\frac{1}{\rho}\right) b$, and hence

$$
\left|\frac{b}{a(a-b)}\right| \leq \frac{1}{1-\frac{1}{\rho}} \cdot \frac{1}{a}=\frac{\rho}{\rho-1} \cdot\left(\frac{\Lambda(a)}{a} / \frac{\Lambda(b)}{b}\right) \cdot \frac{\Lambda(b)}{b \Lambda(a)} .
$$

Since $R \leq a \leq \frac{1}{\rho} b \leq b$, it follows from the monotonicity of $\frac{\Lambda(r)}{r}$ that the second factor is bounded by 1 .

If $\frac{a}{b} \geq \rho$, then $|a-b|=\left(1-\frac{b}{a}\right) a \geq\left(1-\frac{1}{\rho}\right) a$, and hence

$$
\left|\frac{b}{a(a-b)}\right| \leq \frac{1}{1-\frac{1}{\rho}} \frac{b}{a^{2}}=\frac{\rho}{\rho-1} \cdot\left(\frac{\Lambda(a)}{a^{2}} / \frac{\Lambda(b)}{b^{2}}\right) \cdot \frac{\Lambda(b)}{b \Lambda(a)} .
$$

Since $R \leq b \leq \frac{1}{\rho} a \leq a$, the second factor is bounded by 1 , where we used the monotonicity of $\frac{\Lambda(r)}{r^{2}}$. Putting this together we obtain the required estimate.

Next assume that $a, b<0$. The already proved case, applied to $|a|,|b|$, yields

$$
\left|\frac{b}{a(a-b)}\right|=\left|\frac{|b|}{|a|(|a|-|b|)}\right| \leq \frac{\rho}{\rho-1} \cdot \frac{\Lambda(|b|)}{|b| \Lambda(|a|)} .
$$

It remains to consider the case when $a$ and $b$ have different signs. Then, using the same estimates as above, we obtain

$$
\left|\frac{b}{a(a-b)}\right|=\frac{|b|}{|a|(|a|+|b|)} \leq\left\{\begin{array}{cc}
\frac{1}{|a|} & \text { if }|b| \geq|a| \\
\frac{|b|}{|a|^{2}} & \text { if }|b|<|a|
\end{array}\right\} \leq \frac{\Lambda(|b|)}{|b| \Lambda(|a|)}
$$

which finishes the proof. 
3.9 Proposition. Let the following data be given:

- a finite subset I of $\mathbb{N}$;

- a sequence $\xi=\left(\xi_{k}\right)_{k \in I}$ of pairwise distinct and non-zero real numbers;

- a growth function $\Lambda$ and $R_{0}>0$ such that $\frac{\Lambda(r)}{r}$ is either non-increasing or non-decreasing on $\left[R_{0}, \infty\right)$; in the latter case assume, in addition, that $\frac{\Lambda(r)}{r^{2}}$ is decreasing on $\left[R_{0}, \infty\right)$;

- a number $\rho>1$;

- a sequence $\nu=\left(\nu_{k}\right)_{k \in I}$ of real numbers with $\nu_{k} \neq-\xi_{k}, k \in I$;

- an element $n \in I$.

Set

$$
\begin{aligned}
\delta & :=\frac{1}{4\left(2 \frac{\rho^{2}}{\rho-1}+1\right)}, \\
\eta_{k} & :=\xi_{k}+\nu_{k}, \quad k \in I,
\end{aligned}
$$

and

$$
\begin{aligned}
& S_{1}(n):=\sum_{k \in I} \frac{\left|\nu_{k}\right|}{\left|\xi_{k}\right|}, \quad S_{2}(n):=\sum_{\substack{k \in I, k \neq n \\
\frac{\xi_{k}}{\xi_{n}} \in\left(\frac{1}{\rho}, \rho\right)}} \frac{\left|\nu_{k}\right|}{\left|\xi_{k}-\xi_{n}\right|}, \\
& S_{3}(n):=\frac{\left|\nu_{n}\right|}{\left|\xi_{n}\right|} \Lambda\left(\left|\xi_{n}\right|\right) \sum_{k \in I} \frac{1}{\Lambda\left(\left|\xi_{k}\right|\right)}, \quad S_{4}(n):=\left|\nu_{n}\right| \sum_{\substack{k \in I, k \neq n \\
\frac{\xi_{k}}{\xi_{n}} \in\left(\frac{1}{\rho}, \rho\right)}} \frac{1}{\left|\xi_{k}-\xi_{n}\right|}, \\
& T(n):=\frac{\left|\nu_{n}\right|}{\left|\xi_{n}\right|} n_{\xi}\left(\left|\xi_{n}\right|\right), \\
& \Theta(n):=\frac{\log 4}{1-\delta}\left[\frac{\rho}{\rho-1} S_{1}(n)+\rho S_{2}(n)+\frac{\rho}{\rho-1} T(n)+\frac{\rho^{2}}{\rho-1} S_{3}(n)+S_{4}(n)\right], \\
& \widetilde{\Theta}(n):=\frac{\log 4}{1-2 \delta}\left[\frac{\rho}{\rho-1} S_{1}(n)+\rho S_{2}(n)+\frac{\rho}{\rho-1} S_{3}(n)+\rho S_{4}(n)\right] .
\end{aligned}
$$

Assume that

$$
\forall k \in I: \quad \frac{\left|\nu_{n}\right|}{\left|\xi_{k}\right|} \leq \delta, \quad \frac{\left|\nu_{k}\right|}{\left|\xi_{k}\right|} \leq \delta, \quad \frac{\left|\nu_{k}\right|}{s_{\xi}(k)} \leq \delta, \quad\left|\xi_{k}\right| \geq \rho R_{0},
$$

where $s_{\xi}(n)$ is defined as in Definition 2.6.

(i) If $\frac{\Lambda(r)}{r}$ is non-increasing on $\left[R_{0}, \infty\right)$, then

$$
|\log | \prod_{\substack{k \in I \\ k \neq n}} \frac{1-\frac{\eta_{n}}{\eta_{k}}}{1-\frac{\xi_{n}}{\xi_{k}}}|| \leq \Theta(n)
$$


(ii) If $\frac{\Lambda(r)}{r}$ is non-decreasing on $\left[R_{0}, \infty\right)$, then

$$
\left|\log \left(\left|\prod_{\substack{k \in I \\ k \neq n}} \frac{1-\frac{\eta_{n}}{\eta_{k}}}{1-\frac{\xi_{n}}{\xi_{k}}}\right| /\left|\prod_{\substack{k \in I \\ k \neq n}}\left(1-\frac{\nu_{n}}{\eta_{k}}\right)\right|\right)\right| \leq \widetilde{\Theta}(n) .
$$

Proof.

Step 1: rewriting products. For $k \neq n$ we can rewrite

$$
\begin{aligned}
\frac{1-\frac{\eta_{n}}{\eta_{k}}}{1-\frac{\xi_{n}}{\xi_{k}}} & =\frac{\xi_{k}\left(\eta_{k}-\eta_{n}\right)}{\eta_{k}\left(\xi_{k}-\xi_{n}\right)}=1+\frac{\xi_{k}\left(\eta_{k}-\eta_{n}\right)-\eta_{k}\left(\xi_{k}-\xi_{n}\right)}{\eta_{k}\left(\xi_{k}-\xi_{n}\right)} \\
& =1+\frac{\xi_{n} \eta_{k}-\xi_{k} \eta_{n}}{\eta_{k}\left(\xi_{k}-\xi_{n}\right)}=1+\frac{\xi_{n}\left(\eta_{k}-\xi_{k}\right)-\xi_{k}\left(\eta_{n}-\xi_{n}\right)}{\eta_{k}\left(\xi_{k}-\xi_{n}\right)} \\
& =1+\frac{\xi_{n} \nu_{k}-\xi_{k} \nu_{n}}{\eta_{k}\left(\xi_{k}-\xi_{n}\right)} .
\end{aligned}
$$

The latter expression can also be written as

$$
\begin{aligned}
& 1-\frac{\nu_{n}}{\eta_{k}}+\frac{\xi_{n} \nu_{k}-\xi_{k} \nu_{n}+\nu_{n}\left(\xi_{k}-\xi_{n}\right)}{\eta_{k}\left(\xi_{k}-\xi_{n}\right)} \\
& =1-\frac{\nu_{n}}{\eta_{k}}+\frac{\xi_{n}\left(\nu_{k}-\nu_{n}\right)}{\eta_{k}\left(\xi_{k}-\xi_{n}\right)} \\
& =\left(1-\frac{\nu_{n}}{\eta_{k}}\right)\left[1-\left(1-\frac{\nu_{n}}{\eta_{k}}\right)^{-1} \frac{\xi_{n}\left(\nu_{k}-\nu_{n}\right)}{\eta_{k}\left(\xi_{k}-\xi_{n}\right)}\right] .
\end{aligned}
$$

Using (3.17) and (3.18), respectively, we obtain

$$
\begin{aligned}
& \prod_{\substack{k \in I \\
k \neq n}} \frac{1-\frac{\eta_{n}}{\eta_{k}}}{1-\frac{\xi_{n}}{\xi_{k}}}=\prod_{\substack{k \in I \\
k \neq n}}\left[1+\frac{\xi_{n} \nu_{k}-\xi_{k} \nu_{n}}{\eta_{k}\left(\xi_{k}-\xi_{n}\right)}\right], \\
& \frac{\prod_{\substack{k \in I \\
k \neq n}} \frac{1-\frac{\eta_{n}}{\eta_{k}}}{1-\frac{\xi_{n}}{\xi_{k}}}}{\prod_{\substack{k \in I \\
k \neq n}}\left(1-\frac{\nu_{n}}{\eta_{k}}\right)}=\prod_{\substack{k \in I \\
k \neq n}}\left[1-\left(1-\frac{\nu_{n}}{\eta_{k}}\right)^{-1} \frac{\xi_{n}\left(\nu_{k}-\nu_{n}\right)}{\eta_{k}\left(\xi_{k}-\xi_{n}\right)}\right] .
\end{aligned}
$$

Step 2: Remark 3.7 is applicable. Our assumptions imply that, for each $k \in I$,

$$
\begin{aligned}
& \left|\frac{\eta_{k}}{\xi_{k}}\right|=\left|1+\frac{\nu_{k}}{\xi_{k}}\right| \geq 1-\left|\frac{\nu_{k}}{\xi_{k}}\right| \geq 1-\delta, \\
& \left|1-\frac{\nu_{n}}{\eta_{k}}\right| \geq 1-\left|\frac{\xi_{k}}{\eta_{k}}\right| \cdot\left|\frac{\nu_{n}}{\xi_{k}}\right| \geq 1-\frac{1}{1-\delta} \delta=\frac{1-2 \delta}{1-\delta} .
\end{aligned}
$$

Moreover, Lemma 3.8 applied with the growth function $\Lambda(r)=r$ gives

$$
\left|\frac{\xi_{n}}{\xi_{k}\left(\xi_{k}-\xi_{n}\right)}\right| \leq \begin{cases}\frac{\rho}{\rho-1} \frac{1}{\left|\xi_{k}\right|} & \text { if } \frac{\xi_{n}}{\xi_{k}} \notin\left(\frac{1}{\rho}, \rho\right), \\ \frac{\rho}{\left|\xi_{k}-\xi_{n}\right|} & \text { if } \frac{\xi_{n}}{\xi_{k}} \in\left(\frac{1}{\rho}, \rho\right),\end{cases}
$$


and hence

$$
\begin{aligned}
& \left|\frac{\xi_{n} \nu_{k}}{\xi_{k}\left(\xi_{k}-\xi_{n}\right)}\right| \leq \begin{cases}\frac{\rho}{\rho-1} \frac{\left|\nu_{k}\right|}{\left|\xi_{k}\right|} \leq \delta \frac{\rho}{\rho-1} & \text { if } \frac{\xi_{n}}{\xi_{k}} \notin\left(\frac{1}{\rho}, \rho\right), \\
\rho \frac{\left|\nu_{k}\right|}{s_{\xi}(k)} \leq \delta \rho & \text { if } \frac{\xi_{n}}{\xi_{k}} \in\left(\frac{1}{\rho}, \rho\right),\end{cases} \\
& \left|\frac{\xi_{n} \nu_{n}}{\xi_{k}\left(\xi_{k}-\xi_{n}\right)}\right| \leq\left\{\begin{array}{ll}
\frac{\rho}{\rho-1} \frac{\left|\nu_{n}\right|}{\left|\xi_{k}\right|} \leq \delta \frac{\rho}{\rho-1} & \text { if } \frac{\xi_{n}}{\xi_{k}} \notin\left(\frac{1}{\rho}, \rho\right), \\
\rho \frac{\left|\nu_{n}\right|}{s_{\xi}(n)} & \leq \delta \rho
\end{array} \text { if } \frac{\xi_{n}}{\xi_{k}} \in\left(\frac{1}{\rho}, \rho\right) .\right.
\end{aligned}
$$

Both expressions are bounded by $\delta \frac{\rho^{2}}{\rho-1}$ in each case. The definition of $\delta$ yields $\delta \leq \frac{1}{4}$ and $\frac{\rho^{2}}{\rho-1} \leq \frac{1}{8 \delta}$, and thus we obtain

$$
\begin{aligned}
\left|\frac{\xi_{n} \nu_{k}-\xi_{k} \nu_{n}}{\eta_{k}\left(\xi_{k}-\xi_{n}\right)}\right| & =\left|\frac{\xi_{k}}{\eta_{k}}\left(\frac{\xi_{n} \nu_{k}}{\xi_{k}\left(\xi_{k}-\xi_{n}\right)}-\frac{\nu_{n}}{\xi_{k}-\xi_{n}}\right)\right| \\
& \leq \frac{1}{1-\delta}\left(\left|\frac{\xi_{n} \nu_{k}}{\xi_{k}\left(\xi_{k}-\xi_{n}\right)}\right|+\frac{\left|\nu_{n}\right|}{s_{\xi}(n)}\right) \\
& \leq \frac{1}{1-\delta}\left(\delta \frac{\rho^{2}}{\rho-1}+\delta\right) \leq \frac{1}{1-\delta}\left(\frac{1}{8}+\delta\right) \leq \frac{1}{1-\frac{1}{4}}\left(\frac{1}{8}+\frac{1}{4}\right)=\frac{1}{2}
\end{aligned}
$$

and

$$
\begin{aligned}
\left|\left(1-\frac{\nu_{n}}{\eta_{k}}\right)^{-1} \frac{\xi_{n}\left(\nu_{k}-\nu_{n}\right)}{\eta_{k}\left(\xi_{k}-\xi_{n}\right)}\right| & =\left|1-\frac{\nu_{n}}{\eta_{k}}\right|^{-1} \cdot\left|\frac{\xi_{k}}{\eta_{k}}\right| \cdot\left|\frac{\xi_{n} \nu_{k}}{\xi_{k}\left(\xi_{k}-\xi_{n}\right)}-\frac{\xi_{n} \nu_{n}}{\xi_{k}\left(\xi_{k}-\xi_{n}\right)}\right| \\
& \leq \frac{1-\delta}{1-2 \delta} \cdot \frac{1}{1-\delta} \cdot 2 \delta \frac{\rho^{2}}{\rho-1} \leq \frac{1}{1-2 \cdot \frac{1}{4}} \cdot \frac{2}{8}=\frac{1}{2}
\end{aligned}
$$

Therefore we can apply Remark 3.7 to the right-hand sides of (3.19) and (3.20) and obtain the estimates

$$
\begin{aligned}
& |\log | \prod_{\substack{k \in I \\
k \neq n}} \frac{1-\frac{\eta_{n}}{\eta_{k}}}{1-\frac{\xi_{n}}{\xi_{k}}}||=|\log | \prod_{\substack{k \in I \\
k \neq n}}\left[1+\frac{\xi_{n} \nu_{k}-\xi_{k} \nu_{n}}{\eta_{k}\left(\xi_{k}-\xi_{n}\right)}\right]|| \\
& \leq \log 4 \sum_{\substack{k \in I \\
k \neq n}}\left|\frac{\xi_{n} \nu_{k}-\xi_{k} \nu_{n}}{\eta_{k}\left(\xi_{k}-\xi_{n}\right)}\right|=\log 4 \sum_{\substack{k \in I \\
k \neq n}}\left|\frac{\xi_{k}}{\eta_{k}}\right| \cdot\left|\frac{\xi_{n} \nu_{k}}{\xi_{k}\left(\xi_{k}-\xi_{n}\right)}-\frac{\nu_{n}}{\xi_{k}-\xi_{n}}\right| \\
& \leq \frac{\log 4}{1-\delta}\left(\sum_{\substack{k \in I \\
k \neq n}}\left|\frac{\xi_{n} \nu_{k}}{\xi_{k}\left(\xi_{k}-\xi_{n}\right)}\right|+\sum_{\substack{k \in I \\
k \neq n}}\left|\frac{\nu_{n}}{\xi_{k}-\xi_{n}}\right|\right)
\end{aligned}
$$


and

$$
\begin{aligned}
& \left|\log \left(\left|\prod_{\substack{k \in I \\
k \neq n}} \frac{1-\frac{\eta_{n}}{\eta_{k}}}{1-\frac{\xi_{n}}{\xi_{k}}}\right| /\left|\prod_{\substack{k \in I \\
k \neq n}}\left(1-\frac{\nu_{n}}{\eta_{k}}\right)\right|\right)\right| \\
& =|\log | \prod_{\substack{k \in I \\
k \neq n}}\left[1-\left(1-\frac{\nu_{n}}{\eta_{k}}\right)^{-1} \frac{\xi_{k}}{\eta_{k}} \cdot \frac{\xi_{n}\left(\nu_{k}-\nu_{n}\right)}{\xi_{k}\left(\xi_{k}-\xi_{n}\right)}\right]|| \\
& \leq \log 4 \sum_{\substack{k \in I \\
k \neq n}}\left|1-\frac{\nu_{n}}{\eta_{k}}\right|^{-1} \cdot\left|\frac{\xi_{k}}{\eta_{k}}\right| \cdot\left|\frac{\xi_{n} \nu_{k}-\xi_{n} \nu_{n}}{\xi_{k}\left(\xi_{k}-\xi_{n}\right)}\right| \\
& \leq \frac{\log 4}{1-2 \delta}\left(\sum_{\substack{k \in I \\
k \neq n}}\left|\frac{\xi_{n} \nu_{k}}{\xi_{k}\left(\xi_{k}-\xi_{n}\right)}\right|+\sum_{\substack{k \in I \\
k \neq n}}\left|\frac{\xi_{n} \nu_{n}}{\xi_{k}\left(\xi_{k}-\xi_{n}\right)}\right|\right) .
\end{aligned}
$$

Step 3: estimating sums. It is relatively straightforward to estimate the expression that appears as the first sum in both (3.22) and (3.23). To this end we split the summation into two parts: the first inequality in (3.21) gives

$$
\sum_{\substack{k \in I \\ \frac{\xi_{k}}{\xi_{n}} \notin\left(\frac{1}{\rho}, \rho\right)}}\left|\frac{\xi_{n} \nu_{k}}{\xi_{k}\left(\xi_{k}-\xi_{n}\right)}\right| \leq \frac{\rho}{\rho-1} \sum_{\substack{k \in I \\ \frac{\xi_{k}}{\xi_{n}} \notin\left(\frac{1}{\rho}, \rho\right)}} \frac{\left|\nu_{k}\right|}{\left|\xi_{k}\right|} \leq \frac{\rho}{\rho-1} S_{1}(n)
$$

and, by the definition of $S_{2}(n)$, we have

$$
\sum_{\substack{k \in I, k \neq n \\ \frac{\xi_{k}}{\xi_{n}} \in\left(\frac{1}{\rho}, \rho\right)}}\left|\frac{\xi_{n} \nu_{k}}{\xi_{k}\left(\xi_{k}-\xi_{n}\right)}\right| \leq \rho S_{2}(n) .
$$

Next, let us estimate the second sum in (3.22) under the assumption that $\frac{\Lambda(r)}{r}$ is non-increasing. If $\frac{\xi_{k}}{\xi_{n}} \in\left[-\frac{1}{\rho}, \frac{1}{\rho}\right]$, then $\left|\xi_{k}-\xi_{n}\right|=\left|\frac{\xi_{k}}{\xi_{n}}-1\right| \cdot\left|\xi_{n}\right| \geq\left(1-\frac{1}{\rho}\right)\left|\xi_{n}\right|$. Hence

$$
\sum_{\substack{k \in I \\ \frac{\xi_{k}}{\xi_{n}} \in\left[-\frac{1}{\rho}, \frac{1}{\rho}\right]}}\left|\frac{\nu_{n}}{\xi_{k}-\xi_{n}}\right| \leq \frac{\rho}{\rho-1} \frac{\left|\nu_{n}\right|}{\left|\xi_{n}\right|} n_{\xi}\left(\frac{1}{\rho}\left|\xi_{n}\right|\right) \leq \frac{\rho}{\rho-1} T(n) .
$$

If $\frac{\xi_{k}}{\xi_{n}}<-\frac{1}{\rho}$, then $\left|\xi_{k}-\xi_{n}\right| \geq\left|\xi_{k}\right|$ and $\left|\xi_{k}\right| \geq \frac{1}{\rho}\left|\xi_{n}\right| \geq R_{0}$. Hence, using that $\frac{\Lambda(r)}{r}$ is non-increasing on $\left[R_{0}, \infty\right)$ we obtain

$$
\begin{aligned}
\sum_{\substack{k \in I \\
\frac{\xi_{k}}{\xi_{n}}<-\frac{1}{\rho}}}\left|\frac{\nu_{n}}{\xi_{k}-\xi_{n}}\right| & \leq\left|\nu_{n}\right| \sum_{\substack{k \in I \\
\frac{\xi_{k}}{\xi_{n}}<-\frac{1}{\rho}}} \frac{1}{\left|\xi_{k}\right|}=\left|\nu_{n}\right| \sum_{\substack{k \in I \\
\frac{\xi_{k}}{\xi_{n}}<-\frac{1}{\rho}}} \frac{\Lambda\left(\left|\xi_{k}\right|\right)}{\left|\xi_{k}\right|} \cdot \frac{1}{\Lambda\left(\left|\xi_{k}\right|\right)} \\
& \leq\left|\nu_{n}\right| \frac{\Lambda\left(\frac{1}{\rho}\left|\xi_{n}\right|\right)}{\frac{1}{\rho}\left|\xi_{n}\right|} \cdot \sum_{\substack{k \in I \\
\frac{\xi_{k}}{\xi_{n}}<-\frac{1}{\rho}}} \frac{1}{\Lambda\left(\left|\xi_{k}\right|\right)} \leq \rho S_{3}(n) .
\end{aligned}
$$


If $\frac{\xi_{k}}{\xi_{n}} \geq \rho$, then $\left|\xi_{k}-\xi_{n}\right| \geq\left(1-\frac{1}{\rho}\right)\left|\xi_{k}\right|$ and $\left|\xi_{k}\right| \geq \rho\left|\xi_{n}\right| \geq\left|\xi_{n}\right|$. We can again use the monotonicity of $\frac{\Lambda(r)}{r}$ for the estimate

$$
\begin{aligned}
& \sum_{\substack{k \in I \\
\xi_{k}}}\left|\frac{\nu_{n}}{\xi_{k}-\xi_{n}}\right| \leq \frac{\rho}{\rho-1}\left|\nu_{n}\right| \sum_{\substack{k \in I \\
\xi_{k}}} \frac{1}{\left|\xi_{k}\right|}=\frac{\rho}{\rho-1}\left|\nu_{n}\right| \sum_{\substack{k \in I \\
\xi_{k}}} \frac{\Lambda\left(\left|\xi_{k}\right|\right)}{\left|\xi_{k}\right|} \cdot \frac{1}{\Lambda\left(\left|\xi_{k}\right|\right)} \\
& \begin{array}{lll}
\frac{\xi_{k}}{\xi_{n}} \geq \rho & \frac{\xi_{k}}{\xi_{n}} \geq \rho & \frac{\xi_{k}}{\xi_{n}} \geq \rho
\end{array} \\
& \leq \frac{\rho}{\rho-1}\left|\nu_{n}\right| \frac{\Lambda\left(\left|\xi_{n}\right|\right)}{\left|\xi_{n}\right|} \cdot \sum_{\substack{k \in I \\
\frac{\xi_{k}}{\xi_{n}} \geq \rho}} \frac{1}{\Lambda\left(\left|\xi_{k}\right|\right)} \leq \frac{\rho}{\rho-1} S_{3}(n) .
\end{aligned}
$$

By definition

$$
\sum_{\substack{k \in I, k \neq n \\ \frac{\xi_{k}}{\xi_{n}} \in\left(\frac{1}{\rho}, \rho\right)}}\left|\frac{\nu_{n}}{\xi_{k}-\xi_{n}}\right|=S_{4}(n)
$$

and hence

$$
\sum_{\substack{k \in I \\ k \neq n}}\left|\frac{\nu_{n}}{\xi_{k}-\xi_{n}}\right| \leq \frac{\rho}{\rho-1} T(n)+\left(\rho+\frac{\rho}{\rho-1}\right) S_{3}(n)+S_{4}(n)
$$

if $\frac{\Lambda(r)}{r}$ is non-increasing on $\left[R_{0}, \infty\right)$.

Finally, we have to estimate the second sum in (3.23) under the assumption that $\frac{\Lambda(r)}{r}$ is non-decreasing. Since $\frac{\Lambda(r)}{r^{2}}$ is decreasing on $\left[R_{0}, \infty\right)$ in this case by assumption, we can apply Lemma 3.8 with the growth function $\Lambda$, which yields

$$
\sum_{\substack{k \in I \\ \frac{\xi_{k}}{\xi_{n}} \notin\left(\frac{1}{\rho}, \rho\right)}}\left|\frac{\xi_{n} \nu_{n}}{\xi_{k}\left(\xi_{k}-\xi_{n}\right)}\right| \leq \frac{\rho}{\rho-1}\left|\nu_{n}\right| \sum_{\substack{k \in I \\ \frac{\xi_{k}}{\xi_{n}} \notin\left(\frac{1}{\rho}, \rho\right)}} \frac{\Lambda\left(\left|\xi_{n}\right|\right)}{\left|\xi_{n}\right| \Lambda\left(\left|\xi_{k}\right|\right)} \leq \frac{\rho}{\rho-1} S_{3}(n) .
$$

Furthermore,

$$
\sum_{\substack{k \in I, k \neq n \\ \frac{\xi_{k}}{\xi_{n}} \in\left(\frac{1}{\rho}, \rho\right)}}\left|\frac{\xi_{n} \nu_{n}}{\xi_{k}\left(\xi_{k}-\xi_{n}\right)}\right| \leq \rho\left|\nu_{n}\right| \sum_{\substack{k \in I, k \neq n \\ \frac{\xi_{k}}{\xi_{n}} \in\left(\frac{1}{\rho}, \rho\right)}} \frac{1}{\left|\xi_{k}-\xi_{n}\right|}=\rho S_{4}(n) .
$$

Putting these formulae together we obtain the required estimates (3.15) and (3.16).

\subsection{Finishing the proof of Theorem 3.3}

Throughout this subsection, let $\alpha, \beta, \gamma$ and $\Lambda$ be as in Theorem 3.3.

3.10 Remark. In the next subsection (Section 3.3) we show that, for regularly distributed sequences, Hypothesis (B) in Theorem 3.3 can be weakened. Therefore it is important to keep track at which places (B) is used. We mark those places with

3.11 Remark. For the proof we may assume, without loss of generality, that $\rho_{\Lambda}<2$. In fact, since, by Condition (S2), $\alpha$ must grow at least linearly, we can always use $\Lambda(r)=r(\log r)^{2}$, for which we have $\rho_{\Lambda}=1$. Clearly, Condition 
(A) becomes less restrictive if $\Lambda$ is chosen smaller. In particular, if $\Lambda_{1}, \Lambda_{2}$ are two growth functions with $\rho_{\Lambda_{1}}<\rho_{\Lambda_{2}}$, then it follows from Remark 2.2 (iii) that $\frac{\Lambda_{2}(r)}{\Lambda_{1}(r)}$ is increasing for large $r$.

Moreover, note that if $\rho_{\Lambda}<2$, then $\frac{\Lambda(r)}{r^{2}}$ is decreasing for large $r$ again by Remark $2.2($ iii $)$.

The strategy is to cut off the sequences $\alpha$ and $\beta$ at a very large radius $r$, split the product into two parts, apply the estimates for finite sequences from the previous subsection and show that these are uniform in $r$. Then we pass to the whole sequences again.

In order to be able to apply Proposition 3.9, we must make sure that the assumption (3.14) is satisfied. Let $r_{n}$ and $J_{n}(c, \Lambda)$ be defined as in Proposition 3.6 using the sequence $\alpha$ instead of $\xi$, i.e.

$$
r_{n} \Lambda\left(r_{n}\right)=\left|\alpha_{n}\right|, \quad J_{n}(c, \Lambda)=\left\{k \in \mathbb{N}:\left|\alpha_{k}\right| \leq c r_{n}\right\} .
$$

3.12 Lemma. Let $\Lambda$ be a growth function and $R_{0}>0$ such that $\frac{\Lambda(r)}{r}$ is nonincreasing or non-decreasing on $\left[R_{0}, \infty\right)$ and that $\frac{\Lambda(r)}{r^{2}}$ is decreasing on $\left[R_{0}, \infty\right)$. Moreover, let $\delta>0$ and $\rho>1$ be given.

Then there exist $c>0, r_{0} \geq 1$ and $N_{0} \in \mathbb{N}$ such that, for each $n \geq N_{0}$ and $r \geq\left|\alpha_{n}\right|$, the following data satisfy the hypotheses of Proposition 3.9 (i.e. it satisfies (3.14) and $n \in I)$ :

$-I:=\left\{k \in \mathbb{N}: r_{0} \leq\left|\alpha_{k}\right| \leq r\right\} \backslash J_{n}(c, \Lambda) ;$

$-\xi:=\left(\alpha_{k}\right)_{k \in I}$

$-\Lambda$ and $R_{0}$

$-\rho$

$-\nu:=\left(\gamma_{k}\right)_{k \in I}$

$-n$.

Proof. Assumption (A) implies that $\frac{\left|\gamma_{k}\right|}{\left|\alpha_{k}\right|}=\mathrm{O}\left(\frac{1}{\Lambda\left(\left|\alpha_{k}\right|\right)}\right), k \rightarrow \infty$. In particular, $\lim _{k \rightarrow \infty} \frac{\left|\gamma_{k}\right|}{\left|\alpha_{k}\right|}=0$. Moreover, by (3.3), we have

$$
\lim _{k \rightarrow \infty} \frac{\left|\gamma_{k}\right|}{s_{\alpha}(k)} \stackrel{\stackrel{2}{=}}{=} 0
$$

Hence we can choose $r_{0} \geq \rho R_{0}$ such that

$$
\frac{\left|\gamma_{k}\right|}{\left|\alpha_{k}\right|} \leq \delta \quad \text { and } \quad \frac{\left|\gamma_{k}\right|}{s_{\alpha}(k)} \leq \delta \quad \text { for all } k \text { with }\left|\alpha_{k}\right| \geq r_{0}
$$

which shows that the second, third and fourth condition in (3.14) are satisfied. Now set $c_{1}:=\sup _{k \in \mathbb{N}} \frac{\left|\gamma_{k}\right|}{\left|\alpha_{k}\right|} \Lambda\left(\left|\alpha_{k}\right|\right)$, define

$$
c:=\frac{c_{1}}{\delta}
$$

and choose $N_{0} \in \mathbb{N}$ such that

$$
\forall n \geq N_{0}: \quad\left\{k \in \mathbb{N}:\left|\alpha_{k}\right|<r_{0}\right\} \subseteq J_{n}(c, \Lambda) \quad \text { and } \quad n \notin J_{n}(c, \Lambda),
$$


which is possible by items $(v i)$ and $(v)$ in the proof of Proposition 3.6. This shows, in particular, that $n \in I$.

Now let $k \in I$. Then $\left|\alpha_{k}\right|>c r_{n}$ since $k \notin J_{n}(c, \Lambda)$. Remembering that $r_{n} \geq \frac{\left|\alpha_{n}\right|}{\Lambda\left(\left|\alpha_{n}\right|\right)}$ by item (ii) in the proof of Proposition 3.6, we obtain that

$$
\left|\gamma_{n}\right| \leq c_{1} \frac{\left|\alpha_{n}\right|}{\Lambda\left(\left|\alpha_{n}\right|\right)} \leq c_{1} r_{n}<\frac{c_{1}}{c}\left|\alpha_{k}\right|=\delta\left|\alpha_{k}\right|,
$$

which proves the first condition in (3.14).

In order to proceed with the proof of Theorem 3.3, choose $\rho>1$ such that the condition in (3.4) holds.

Define $\delta$ as in (3.13). Then $\delta>0$, and we can use $\rho$ and $\delta$ in Lemma 3.12. Note that the assumption on $\Lambda$ in this lemma is satisfied because of Remark 3.11. If we use the data from Lemma 3.12 in Proposition 3.9, then we obtain the bounds in (3.15) or (3.16), depending on the monotonicity of $\frac{\Lambda(r)}{r}$. Of course, these bounds depend on $r$ because the set $I$ in Lemma 3.12 depends on $r$; let us write $I(n ; r)$ instead of $I$ in the following in order to make this explicit. Moreover, we write

$$
\Theta(n ; r), T(n ; r) \text { etc. }
$$

for the expressions in Proposition 3.9.

3.13 Lemma. The following relations hold:

$$
\vartheta:=\sup _{n \geq N_{0}} \sup _{\substack{r \in \mathbb{R} \\ r \geq\left|\alpha_{n}\right|}} \Theta(n ; r)<\infty, \quad \tilde{\vartheta}:=\sup _{n \geq N_{0}} \sup _{\substack{r \in \mathbb{R} \\ r \geq\left|\alpha_{n}\right|}} \widetilde{\Theta}(n ; r)<\infty .
$$

Proof. Set $c_{1}:=\sup _{k \in \mathbb{N}} \frac{\left|\gamma_{k}\right|}{\left|\alpha_{k}\right|} \Lambda\left(\left|\alpha_{k}\right|\right)$, which is finite by Hypothesis (A). Then we have the following $r$-independent bounds (o and O notation is for $n \rightarrow \infty$ ):

$$
\begin{aligned}
& S_{1}(n ; r)=\sum_{k \in I(n ; r)}\left|\frac{\gamma_{k}}{\alpha_{k}}\right| \leq c_{1} \sum_{k \in I(n ; r)} \frac{1}{\Lambda\left(\left|\alpha_{k}\right|\right)} \leq c_{1} \sum_{\substack{k \in \mathbb{N} \\
\left|\alpha_{k}\right|>c r_{n}}} \frac{1}{\Lambda\left(\left|\alpha_{k}\right|\right)}=\mathrm{o}(1),
\end{aligned}
$$

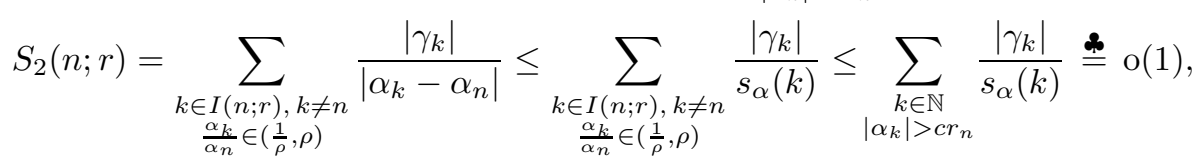

$$
\begin{aligned}
& S_{3}(n ; r)=\frac{\left|\gamma_{n}\right|}{\left|\alpha_{n}\right|} \Lambda\left(\left|\alpha_{n}\right|\right) \sum_{k \in I(n ; r)} \frac{1}{\Lambda\left(\left|\alpha_{k}\right|\right)} \leq c_{1} \sum_{\substack{k \in \mathbb{N} \\
\left|\alpha_{k}\right|>c r_{n}}} \frac{1}{\Lambda\left(\left|\alpha_{k}\right|\right)}=\mathrm{o}(1), \\
& S_{4}(n ; r)=\left|\gamma_{n}\right| \sum_{\substack{k \in I(n ; r), k \neq n \\
\frac{\alpha_{k}}{\alpha_{n}} \in\left(\frac{1}{\rho}, \rho\right)}} \frac{1}{\left|\alpha_{k}-\alpha_{n}\right|} \leq\left|\gamma_{n}\right| \cdot r_{\alpha}(\rho, n) \frac{1}{s_{\alpha}(n)} \stackrel{\text { ॥ }}{=} \mathrm{O}(1), \\
& T(n ; r)=\frac{\left|\gamma_{n}\right|}{\left|\alpha_{n}\right|} n_{\alpha}\left(\left|\alpha_{n}\right|\right) \leq c_{1} \frac{n_{\alpha}\left(\left|\alpha_{n}\right|\right)}{\Lambda\left(\left|\alpha_{n}\right|\right)}=\mathrm{O}(1) ;
\end{aligned}
$$

the last estimate follows from Lemma 2.9. 
3.14 Remark. If we slightly strengthen (3.4) to $\frac{\left|\gamma_{n}\right|}{s_{\alpha}(n)}=\mathrm{o}\left(\frac{1}{r_{\alpha}(\rho, n)}\right)$ and assume, in addition, that $n_{\alpha}(r)=\mathrm{o}(\Lambda(r))$, then we may assert in the above lemma that

$$
\lim _{n \rightarrow \infty} \sup _{\substack{r \in \mathbb{R} \\ r \geq\left|\alpha_{n}\right|}} \Theta(n ; r)=0 \quad \text { and } \quad \lim _{n \rightarrow \infty} \sup _{\substack{r \in \mathbb{R} \\ r \geq\left|\alpha_{n}\right|}} \widetilde{\Theta}(n ; r)=0,
$$

respectively.

3.15 Remark. The estimates used for $S_{2}(n ; r)$ and $S_{4}(n ; r)$ may seem rather careless. However, for wildly behaving sequences $\alpha$, they can be sharp. Such sequences are for example constructed by mixing lumps of points of the following two kinds leaving sufficiently large empty intervals in between them:

- Type 1:

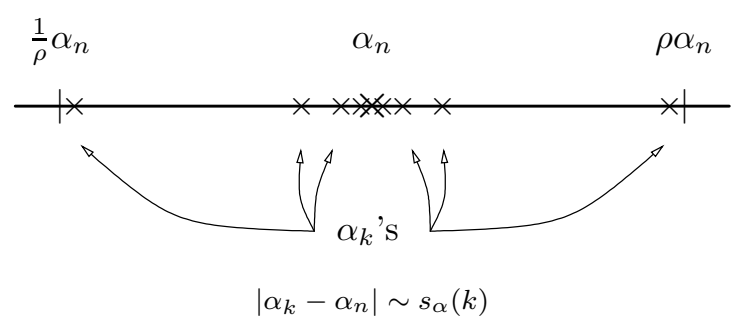

- Type 2:

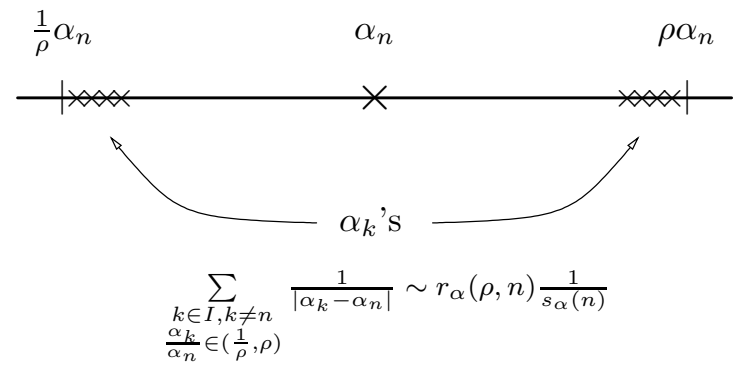

Having available the uniform estimate from Lemma 3.13, we can now complete the proof of Theorem 3.3. We only have to take care of the following slight subtlety.

3.16 Remark. Let $l, m \in \mathbb{N}$ be such that $\alpha_{l}<\alpha_{m}$ are two consecutive members of the point set $\left\{\alpha_{k}: k \in \mathbb{N}\right\}$, and assume that $\left|\alpha_{l}\right|,\left|\alpha_{m}\right| \geq r_{0}$. By our choice of $\delta$ in (3.13), it follows from the second inequality in (3.24) that $\frac{\left|\gamma_{k}\right|}{s_{\alpha}(k)}<\frac{1}{2}$ for $k=l$ and $k=m$, and hence

$$
\beta_{l}<\beta_{m}, \quad \alpha_{l}<\beta_{m}, \quad \beta_{l}<\alpha_{m},
$$

i.e. the pair $\left\{\alpha_{l}, \beta_{l}\right\}$ is separated from the pair $\left\{\alpha_{m}, \beta_{m}\right\}$. This implies that, for each sufficiently large $r$ (where ' $\triangle$ ' denotes the symmetric difference),

$$
\#\left(\left\{k \in \mathbb{N}:\left|\alpha_{k}\right| \leq r\right\} \triangle\left\{k \in \mathbb{N}:\left|\beta_{k}\right| \leq r\right\}\right) \leq 2 .
$$


Let $r>r_{0}$ be given and assume that $\left\{k \in \mathbb{N}: r_{0} \leq\left|\alpha_{k}\right| \leq r\right\} \neq \emptyset$. Then we define $k_{+}$as the index with

$$
\alpha_{k_{+}}=\max _{\alpha_{k} \leq r} \alpha_{k} .
$$

If the set $\left\{k \in \mathbb{N}: \alpha_{k}>r\right\}$ is non-empty, we define $k_{+}^{\prime}$ as the index with

$$
\alpha_{k_{+}^{\prime}}=\min _{\alpha_{k}>r} \alpha_{k}
$$

and say that ' $k_{+}^{\prime}$ exists'. If $\left\{k \in \mathbb{N}: \alpha_{k}>r\right\}=\emptyset$, we say that $k_{+}^{\prime}$ does not exist. With this notation we set

$$
Q_{r}^{+}(z):= \begin{cases}\left(1-\frac{z}{\beta_{k_{+}}}\right)^{-1}, & \beta_{k_{+}}>r \\ 1-\frac{z}{\beta_{k_{+}^{\prime}}}, & k_{+}^{\prime} \text { exists and } \beta_{k_{+}^{\prime}} \leq r \\ 1, & \text { otherwise. }\end{cases}
$$

Indices $k_{-}, k_{-}^{\prime}$ and a function $Q_{r}^{-}(z)$ are defined in the similar way, taking care of the left endpoint of the interval $[-r, r]$.

Proof of Theorem 3.3: the case when $\frac{\Lambda(r)}{r}$ is non-increasing. Let $n \geq N_{0}$ and $r \geq\left|\alpha_{n}\right|$. Then, using (3.25), we can write

$$
\left\{k \in \mathbb{N}:\left|\alpha_{k}\right| \leq r\right\}=J_{n}(c, \Lambda) \cup\left[\left\{k \in \mathbb{N}: r_{0} \leq\left|\alpha_{k}\right| \leq r\right\} \backslash J_{n}(c, \Lambda)\right],
$$

and this is a disjoint union. In accordance with the paragraph before Lemma 3.13 , we denote the second set in this union by $I(n ; r)$. Hence

$$
\begin{aligned}
& \frac{-\frac{1}{\beta_{n}} \prod_{\substack{\left|\beta_{k}\right| \leq r \\
k \neq n}}\left(1-\frac{\beta_{n}}{\beta_{k}}\right)}{-\frac{1}{\alpha_{n}} \prod_{\substack{\left|\alpha_{k}\right| \leq r \\
k \neq n}}\left(1-\frac{\alpha_{n}}{\alpha_{k}}\right)}=\prod_{k \in J_{n}(c, \Lambda)} \frac{1-\frac{\beta_{n}}{\beta_{k}}}{1-\frac{\alpha_{n}}{\alpha_{k}}} \times \frac{\prod_{\substack{k \in I(n ; r) \\
k \neq n}}\left(1-\frac{\beta_{n}}{\beta_{k}}\right)}{\prod_{\substack{k \in I(n ; r) \\
k \neq n}}\left(1-\frac{\alpha_{n}}{\alpha_{k}}\right)} \times \\
& \times \frac{\alpha_{n}}{\beta_{n}} \times Q_{r}^{+}\left(\beta_{n}\right) Q_{r}^{-}\left(\beta_{n}\right) .
\end{aligned}
$$

If $r \rightarrow \infty$, then the left-hand side tends to $\frac{P_{\beta}^{\prime}\left(\beta_{n}\right)}{P_{\alpha}^{\prime}\left(\alpha_{n}\right)}$, cf. (3.6). The first and the third factors on the right-hand side do not depend on $r$. By Proposition 3.9, the second factor remains bounded from above and away from zero with the bounds $\exp \vartheta_{n}$ and $\exp \left(-\vartheta_{n}\right)$, respectively, where

$$
\vartheta_{n}:=\sup _{\substack{r \in \mathbb{R} \\ r \geq\left|\alpha_{n}\right|}} \Theta(n ; r)
$$

which is finite and uniformly bounded in $n$ by Lemma 3.13. The last factor tends to 1 . We conclude that

$$
\left|\prod_{k \in J_{n}(c, \Lambda)} \frac{1-\frac{\beta_{n}}{\beta_{k}}}{1-\frac{\alpha_{n}}{\alpha_{k}}}\right| e^{-\vartheta_{n}} \frac{\left|\alpha_{n}\right|}{\left|\beta_{n}\right|} \leq\left|\frac{P_{\beta}^{\prime}\left(\beta_{n}\right)}{P_{\alpha}^{\prime}\left(\alpha_{n}\right)}\right| \leq\left|\prod_{k \in J_{n}(c, \Lambda)} \frac{1-\frac{\beta_{n}}{\beta_{k}}}{1-\frac{\alpha_{n}}{\alpha_{k}}}\right| e^{\vartheta_{n}} \frac{\left|\alpha_{n}\right|}{\left|\beta_{n}\right|} .
$$


Now let $n$ tend to $\infty$. The last factors on the left- and the right-hand sides tend to 1 . The respective second factors remain bounded, namely on the right-hand side by $e^{\vartheta}$ from above and on the left-hand side by $e^{-\vartheta}$ from below; for the definition of $\vartheta$ see Lemma 3.13. By Proposition 3.6, the first factor remains bounded from above and away from zero.

3.17 Remark. Maybe it is good to pause and review the proof (thereby also explain the necessity to be precise about constants in O-estimates).

For each fixed $n$ we cut the product into two pieces. In order to have a bound of the partial product corresponding to large indices which is uniform in $n$, we use some uniform estimate of the product by the corresponding sum (we decided to use the ' $\log 4$ '-estimate in Remark 3.7). Application of this uniform estimate is, however, only legitimate if we cut at a point that is so large that (3.14) holds.

The earliest legitimate cutting point depends on $n$. Since the perturbation $\gamma$ need not be bounded, it will in general increase to infinity with $n$. And now Proposition 3.6 and (3.26) come into play, which say that we can control beginning sections of the (n-dependent) product $\frac{P_{\beta}^{\prime}\left(\beta_{n}\right)}{P_{\alpha}^{\prime}\left(\alpha_{n}\right)}$ which are getting longer sufficiently fast.

Proof of Theorem 3.3: the case when $\frac{\Lambda(r)}{r}$ is non-decreasing. Using the same argument as in the previous part of the proof with (3.16) instead of (3.15) and $\tilde{\vartheta}$ instead of $\vartheta$ we obtain that the quotient

$$
\frac{\left|P_{\beta}^{\prime}\left(\beta_{n}\right)\right|}{\left|P_{\alpha}^{\prime}\left(\alpha_{n}\right)\right|} /\left|\prod_{k \notin J_{n}(c, \Lambda)}\left(1-\frac{\gamma_{n}}{\beta_{k}}\right)\right|
$$

is bounded from above and away from zero uniformly in $n$.

The limit $\lim _{n \rightarrow \infty} \prod_{k \notin J_{n}(c, \Lambda)}\left(1-\frac{z}{\beta_{k}}\right)$ exists locally uniformly on $\mathbb{C}$ and equals 1. Since $\frac{\Lambda(r)}{r}$ is non-decreasing, Condition (A) implies that $\gamma$ is bounded. Thus

$$
\lim _{n \rightarrow \infty} \prod_{k \notin J_{n}(c, \Lambda)}\left(1-\frac{\gamma_{n}}{\beta_{k}}\right)=1
$$

which proves the assertion also in this case.

\subsection{Supplements: regularly distributed sequences}

For regularly distributed sequences $\alpha$ we have more control over $s_{\alpha}$ and $r_{\alpha}$. In addition, we can give better estimates for $S_{2}(n)$ and $S_{4}(n)$ from Proposition 3.9 since irregular behaviour as in Remark 3.15 is ruled out.

The conditions required in Theorem 3.3 do not depend on the arrangement of $\alpha$ as long as $\beta$ is arranged in the same way. In the theorems below, however, we need to refer to a specific arrangement of $\alpha$. For this we need one more notation. Let $\alpha \in \mathbb{S}$ and consider the (finite or infinite) subsequence $\alpha^{+}$of $\alpha$ consisting of all positive elements of $\alpha$ arranged increasingly as in Definition 3.1. For each $n$ such that the term $\alpha_{n}^{+}$exists, let $\chi^{+}(n)$ be the unique index with $\alpha_{n}^{+}=\alpha_{\chi^{+}(n)}$. If $\alpha^{+}$is an infinite sequence, then $\chi^{+}$is a bijective map from $\mathbb{N}$ onto $\left\{n \in \mathbb{N}: \alpha_{n}>0\right\}$. Let $\nu^{+}:\left\{n \in \mathbb{N}: \alpha_{n}>0\right\} \rightarrow \mathbb{N}$ be its inverse. Note 
that $\alpha_{n}=\alpha_{\nu^{+}(n)}^{+}$if $\alpha_{n}>0$. Similar notation is used for the subsequence of negative terms of $\alpha$.

First, we assume only a mild regularity property of $\alpha$.

3.18 Theorem. Let $\alpha$ and $\Lambda$ be given as in Theorem 3.3. Further, let $\beta \in \mathbb{S}$ be a small perturbation of $\alpha$ in the sense that $\gamma:=\beta-\alpha$ satisfies (A) from Theorem 3.3 and the following conditions.

(C1) If $\alpha$ contains infinitely many positive terms, then there exists a growth function $\lambda^{+}$such that $\alpha^{+}$has finite upper and positive lower $\lambda^{+}$densities.

If $\alpha$ contains infinitely many negative terms, then there exists a growth function $\lambda^{-}$such that $\alpha^{-}$has finite upper and positive lower $\lambda^{-}$densities.

$$
\frac{\left|\gamma_{\chi^{+}(n)}\right|}{s_{\alpha^{+}}(n)}=\mathrm{O}\left(\frac{1}{n}\right), \quad \frac{\left|\gamma_{\chi^{-}(n)}\right|}{s_{\alpha^{-}}(n)}=\mathrm{O}\left(\frac{1}{n}\right), \quad n \rightarrow \infty
$$

Then (3.5) holds.

The condition (3.27) is of course only a minor weakening of (3.3), but the condition (3.4) can be dropped (in fact, it holds automatically).

Proof of Theorem 3.18. We have to check all places where Condition (B) was used (which were marked with

(1) Proof of Lemma 3.12: Clearly, (3.27) implies that $\lim _{k \rightarrow \infty} \frac{\left|\gamma_{k}\right|}{s_{\alpha}(k)}=0$.

(2) Choice of $\rho$ : Choose $\rho>1$ arbitrarily.

(3) Proof of Lemma 3.13: We have to provide suitable bounds for $S_{2}(n ; r)$ and $S_{4}(n ; r)$. We consider the case when $n$ ranges over those indices with $\alpha_{n}>0$; the set of indices $n$ with $\alpha_{n}<0$ is treated in the same way.

If the sequence $\alpha$ contains only finitely many positive elements, it is clear that $S_{2}(n ; r)$ and $S_{4}(n ; r)$ remain bounded. Hence, assume that $\alpha^{+}$is an infinite sequence. Let $n \in \mathbb{N}$ such that $\alpha_{n}>0$. Then, clearly,

$$
\left\{\alpha_{k}: \frac{\alpha_{k}}{\alpha_{n}} \in\left(\frac{1}{\rho}, \rho\right)\right\}=\left\{\alpha_{l}^{+}: \frac{\alpha_{l}^{+}}{\alpha_{\nu^{+}(n)}^{+}} \in\left(\frac{1}{\rho}, \rho\right)\right\}
$$

and this shows that $r_{\alpha}(\rho, n)=r_{\alpha^{+}}\left(\rho, \nu^{+}(n)\right)$. Unless $\nu^{+}(n)=1$, we also have $s_{\alpha}(n)=s_{\alpha^{+}}\left(\nu^{+}(n)\right)$.

Using (3.27) and Lemma 2.7 we obtain (with $\left.c:=\sup _{n \in \mathbb{N}} \frac{\left|\gamma_{\chi}+(n)\right|}{s_{\alpha}(n)} n\right)$

$$
\begin{aligned}
& S_{2}(n ; r) \leq \sum_{\substack{k \in \mathbb{N} \\
\frac{\alpha_{k}}{\alpha_{n}} \in\left(\frac{1}{\rho}, \rho\right)}} \frac{\left|\gamma_{k}\right|}{s_{\alpha}(k)}=\sum_{\substack{\alpha_{l}^{+} \\
\frac{\alpha^{+}}{\alpha_{\nu^{+}(n)}^{+}} \in\left(\frac{1}{\rho}, \rho\right)}} \frac{\left|\gamma_{\chi^{+}(l)}\right|}{s_{\alpha^{+}}(l)} \leq c \sum_{\substack{l \in \mathbb{N} \\
\frac{\alpha_{l}^{+}}{\alpha_{\nu^{+}(n)}^{+}} \in\left(\frac{1}{\rho}, \rho\right)}} \frac{1}{l}=\mathrm{O}(1), \\
& S_{4}(n ; r) \leq \frac{\left|\gamma_{n}\right| \cdot r_{\alpha}(\rho, n)}{s_{\alpha}(n)}=\frac{\left|\gamma_{\chi^{+}\left(\nu^{+}(n)\right)}\right|}{s_{\alpha^{+}}\left(\nu^{+}(n)\right)} \cdot r_{\alpha^{+}}\left(\rho, \nu^{+}(n)\right) \lesssim \frac{1}{\nu^{+}(n)} \cdot \nu^{+}(n)=1
\end{aligned}
$$

as $n \rightarrow \infty$ uniformly in $r$. 
Second, we assume that $\alpha$ behaves quite regularly. For the definition of the standard sequence $\lambda$ corresponding to a growth function $\lambda$ see Section 2.2.

3.19 Theorem. Let $\alpha$ and $\Lambda$ be given as in Theorem 3.3. Further, let $\beta \in \mathbb{S}$ be a small perturbation of $\alpha$ in the sense that $\gamma:=\beta-\alpha$ satisfies (A) from Theorem 3.3 and the following conditions.

(D1) If $\alpha$ contains infinitely many positive terms, then there exists a growth function $\lambda^{+}$such that $\alpha^{+}=\lambda^{+}$.

If $\alpha$ contains infinitely many negative terms, then there exists a growth function $\lambda^{-}$such that $\alpha^{-}=-\lambda^{-}$.

$$
\left|\gamma_{\chi^{+}(n)}\right|=\mathrm{O}\left(\frac{\alpha_{n}^{+}}{n \log n}\right), \quad\left|\gamma_{\chi^{-}(n)}\right|=\mathrm{O}\left(\frac{\left|\alpha_{n}^{-}\right|}{n \log n}\right), \quad n \rightarrow \infty .
$$

Then (3.5) holds.

Proof. Also here we just have to check the places with a. Again, we restrict the explicit proof to the sequence $\alpha^{+}$and to the case when this sequence is infinite.

(1) Proof of Lemma 3.12: Using the first formula in Lemma 2.12 and (D2) we obtain

$$
\frac{\gamma_{n}^{+}}{s_{\alpha^{+}}(n)} \lesssim \frac{\alpha_{n}^{+}}{n \log n} \cdot \frac{n}{\alpha_{n}^{+}}=\frac{1}{\log n}=\mathrm{o}(1), \quad n \rightarrow \infty .
$$

(2) Choice of $\rho$ : Choose $\rho>1$ arbitrarily.

(3) Proof of Lemma 3.13: For $n \in\left\{l \in \mathbb{N}: \alpha_{l}>0\right\}$ and $r>0$, we can use the second formula in Lemma 2.12 to estimate the following sum:

$$
\begin{aligned}
\sum_{\substack{k \in I(n ; r), k \neq n \\
\frac{\alpha_{k}}{\alpha_{n}} \in\left(\frac{1}{\rho}, \rho\right)}} \frac{1}{\left|\alpha_{k}-\alpha_{n}\right|} & \leq \sum_{\substack{k \in \mathbb{N}, k \neq n \\
\frac{\alpha_{k}}{\alpha_{n}} \in\left(\frac{1}{\rho}, \rho\right)}} \frac{1}{\left|\alpha_{k}-\alpha_{n}\right|}=\sum_{\substack{l \in \mathbb{N}, l \neq \nu^{+}(n) \\
\frac{\alpha_{l}^{+}}{\alpha^{+}}} \in\left(\frac{1}{\left.\nu^{+}, \rho\right)}\right)} \frac{1}{\left|\alpha_{l}^{+}-\alpha_{\nu^{+}(n)}^{+}\right|} \\
& \leq C \frac{\nu^{+}(n) \log \left(\nu^{+}(n)\right)}{\alpha_{\nu^{+}(n)}^{+}}
\end{aligned}
$$

with some $C>0$ independent of $n$. This together with (D2) yields

$$
S_{4}(n ; r)=\left|\gamma_{\chi^{+}\left(\nu^{+}(n)\right)}\right| \sum_{\substack{k \in I(n ; r), k \neq n \\ \frac{\alpha_{k}}{\alpha_{n}} \in\left(\frac{1}{\rho}, \rho\right)}} \frac{1}{\left|\alpha_{k}-\alpha_{n}\right|}=\mathrm{O}(1)
$$

for $n \rightarrow \infty$ uniformly in $r$. For $S_{2}(n ; r)$ we can estimate

$$
\begin{aligned}
& S_{2}(n ; r)=\sum_{\substack{k \in I(n ; r), k \neq n \\
\frac{\alpha_{k}}{\alpha_{n}} \in\left(\frac{1}{\rho}, \rho\right)}}\left|\frac{\gamma_{k}}{\alpha_{k}-\alpha_{n}}\right| \leq \max _{\substack{k \in I(n ; r), k \neq n \\
\frac{\alpha_{k}}{\alpha_{n}} \in\left(\frac{1}{\rho}, \rho\right)}}\left|\gamma_{k}\right| \cdot \sum_{\substack{k \in I(n ; r), k \neq n \\
\frac{\alpha_{k}}{\alpha_{n}} \in\left(\frac{1}{\rho}, \rho\right)}} \frac{1}{\left|\alpha_{k}-\alpha_{n}\right|} \\
& \leq \max _{l \in \mathbb{N}, l \neq \nu^{+}(n)}\left|\gamma_{\chi^{+}(l)}\right| \cdot C \frac{\nu^{+}(n) \log \left(\nu^{+}(n)\right)}{\alpha_{\nu^{+}(n)}^{+}} \text {. } \\
& \frac{\alpha_{l}^{+}}{\alpha_{\nu}^{+}(n)} \in\left(\frac{1}{\rho}, \rho\right)
\end{aligned}
$$


In order to estimate the maximum, let $l \in \mathbb{N}$ such that $\frac{\alpha_{l}^{+}}{\alpha_{\nu+(n)}^{+}} \in\left(\frac{1}{\rho}, \rho\right)$. Then $\alpha_{l}^{+}>\frac{1}{\rho} \alpha_{\nu^{+}(n)}^{+}$and hence, by Lemma $2.11(i i)$ and Remark $2.2(i)$,

$$
l>n_{\alpha^{+}}\left(\frac{1}{\rho} \alpha_{\nu^{+}(n)}^{+}\right) \geq\left\lfloor\lambda^{+}\left(\frac{1}{\rho} \alpha_{\nu^{+}(n)}^{+}\right)\right\rfloor \gtrsim \lambda^{+}\left(\alpha_{\nu^{+}(n)}^{+}\right)=\nu^{+}(n),
$$

which gives (with some $C^{\prime}>0$ )

$$
\left|\gamma_{\chi^{+}(l)}\right| \leq C^{\prime} \frac{\alpha_{l}^{+}}{l \log l} \leq C^{\prime} \frac{\rho \alpha_{\nu^{+}(n)}^{+}}{l \log l} \lesssim \frac{\alpha_{\nu^{+}(n)}^{+}}{\nu^{+}(n) \log \left(\nu^{+}(n)\right)}
$$

This together with (3.28) implies that $S_{2}(n ; r)$ is bounded uniformly in $n$ and $r$.

Under slightly stronger assumptions on $\Lambda, \lambda^{+}$and $\lambda^{-}$one can even drop Condition (D2) as the following corollary shows.

3.20 Corollary. Let $\alpha$ and $\Lambda$ be given as in Theorem 3.3. Further, let $\beta \in \mathbb{S}$ be a small perturbation of $\alpha$ in the sense that $\gamma:=\beta-\alpha$ satisfies (A) from Theorem 3.3 and the following condition.

(E) If $\alpha$ contains infinitely many positive terms, then there exists a growth function $\lambda^{+}$such that $\alpha^{+}=\lambda^{+}$, that $\frac{\lambda^{+}(r)}{\Lambda(r)}$ is non-increasing for large $r$ and that either $\rho_{\lambda^{+}}>0$ or $\frac{\log \lambda^{+}(r)}{\log r}$ is non-increasing for large $r$.

If $\alpha$ contains infinitely many negative terms, then there exists a growth function $\lambda^{-}$such that $\alpha^{-}=-\lambda^{-}$, that $\frac{\lambda^{-}(r)}{\Lambda(r)}$ is non-increasing for large $r$ and that either $\rho_{\lambda^{-}}>0$ or $\frac{\log \lambda^{-}(r)}{\log r}$ is non-increasing for large $r$.

Then (3.5) holds.

3.21 Remark. As already mentioned in Remark 2.14 the assumptions in (E) are satisfied in most cases if $\alpha^{+}$and $\alpha^{-}$are standard sequences, cf. that remark for more details.

In particular, consider the situation that either $\alpha^{-}$is the empty sequence (i.e. $\alpha_{n}>0$ for all $n \in \mathbb{N}$ ) or that $\alpha^{-}=-\alpha^{+}$(i.e. the set $\left\{\alpha_{n}: n \in \mathbb{N}\right\}$ is symmetric). Moreover, assume that $\alpha^{+}$is a standard sequence: $\alpha^{+}=\lambda^{+}=: \AA$ and that either $\rho_{\lambda}>0$ or $\frac{\log \lambda(r)}{\log r}$ is non-increasing for large $r$. In this case we can choose $\Lambda:=\lambda(r) \cdot \log \lambda(r) \cdot(\log \log \lambda(r))^{2}$ for large $r$. Then Condition (E) is satisfied and $\alpha$ is of convergence class with respect to $\Lambda$ since

$$
\sum_{n \in \mathbb{N}} \frac{1}{\Lambda\left(\grave{\circ}_{n}\right)}=\sum_{n \in \mathbb{N}} \frac{1}{n \log n \cdot(\log \log n)^{2}}<\infty .
$$

Hence, if $\gamma$ satisfies Condition (A) from Theorem 3.3, i.e.

$$
\left|\gamma_{\chi^{ \pm}(n)}\right|=\mathrm{O}\left(\frac{\alpha_{n}^{+}}{n \log n \cdot(\log \log n)^{2}}\right), \quad n \rightarrow \infty
$$

then (3.5) holds. 
3.22 Example. In order to illustrate the power of this supplement, let us reconsider the example already discussed in Remark 3.5. That is

$$
\alpha_{n}:=n^{\sigma} \quad \text { with } \sigma>1, \quad \Lambda(r):=r^{\frac{1}{\sigma}} \log r \cdot(\log \log r)^{2}, \quad \text { for large } r .
$$

Then Corollary 3.20 (see also Remark 3.21) states that

$$
\frac{\left|\gamma_{n}\right|}{n^{\sigma-1}}=\mathrm{O}\left(\frac{1}{\log n \cdot(\log \log n)^{2}}\right)
$$

is already enough to have (3.5). Theorem 3.18 requires

$$
\frac{\left|\gamma_{n}\right|}{n^{\sigma-1}}=\mathrm{O}\left(\frac{1}{n}\right)
$$

and Theorem 3.3 even requires

$$
\sum_{n=1}^{\infty} \frac{\left|\gamma_{n}\right|}{n^{\sigma-1}}<\infty
$$

This comparison also reflects the fact that the regularity assumption in Theorem 3.18 is very weak, whereas the one in Theorem 3.19 is quite strong.

One can also consider a linearly growing sequence $\alpha_{n}$. In this case the sequence must be symmetric because of Condition (S2) in Definition 3.1. Consider $\alpha_{n}$ such that $\alpha_{n}^{+}=n, \alpha_{n}^{-}=-n, n \in \mathbb{N}$. Then Corollary 3.20 (see also Remark 3.21) implies that

$$
\left|\gamma_{\chi^{+}(n)}\right|=\mathrm{O}\left(\frac{1}{\log n \cdot(\log \log n)^{2}}\right), \quad\left|\gamma_{\chi^{-}(n)}\right|=\mathrm{O}\left(\frac{1}{\log n \cdot(\log \log n)^{2}}\right)
$$

is sufficient for (3.5) to hold.

We note that one can apply the theorems also to very sparse sequences like exponentially growing ones.

\subsection{Symmetry of conditions}

We close this section with a general note on the nature of the conditions appearing in our results.

In Theorems 3.3, 3.18 and 3.19 we consider $\beta$ as a perturbation of $\alpha$ by $\gamma$, and the conditions (A), (B) etc. relate the perturbation $\gamma$ to the sequence $\alpha$. Strictly speaking we must therefore say that the ordered pair $(\alpha, \beta)$ satisfies the given conditions.

Actually, Theorems 3.3 and 3.18 are symmetric in $\alpha$ and $\beta$ as the following proposition shows.

3.23 Proposition. Let $\alpha, \beta \in \mathbb{S}$ and assume that the hypotheses of one of Theorems 3.3 and 3.18 are fulfilled for the pair $(\alpha, \beta)$. Then also the pair $(\beta, \alpha)$ satisfies the corresponding hypotheses.

Proof. It follows from (3.2) that $\lim _{n \rightarrow \infty} \frac{\beta_{n}}{\alpha_{n}}=1$. Hence $\Lambda\left(\left|\alpha_{n}\right|\right) \asymp \Lambda\left(\left|\beta_{n}\right|\right)$ by Remark $2.2(i)$, and we see that (3.1) holds for $\beta$. Moreover, also (3.2) holds with $\beta$ and $-\gamma$ in place of $\alpha$ and $\gamma$. For sufficiently large $n$, the numbers $\alpha_{n}$ 
and $\beta_{n}$ have the same sign. Remembering Lemma 2.5 we thus obtain that $(\mathrm{C} 1)$ also holds for $\beta$ (with the same growth functions $\lambda^{+}$and $\lambda^{-}$).

For the conditions in (B) and $(\mathrm{C} 2)$ it is not so obvious that the roles of $\alpha$ and $\beta$ can be exchanged. First we investigate the relation between $s_{\alpha}$ and $s_{\beta}$. Let us show that $s_{\alpha}(n) \lesssim s_{\beta}(n)$. Assume that $\alpha$ and $\gamma$ satisfy (B) or (C2). Let us consider positive $\beta_{n}$ and assume that there are infinitely many positive $\beta_{k}$ and hence also infinitely many positive $\alpha_{k}$. Choose $n_{0} \in \mathbb{N}$ such that

$$
\frac{\left|\gamma_{k}\right|}{s_{\alpha}(k)} \leq \frac{1}{4}, \quad k \geq n_{0}
$$

and let $n_{1}$ be such that $\beta_{n_{1}} \geq \beta_{k}$ for all $k<n_{0}$ and $\beta_{n_{1}}>0$. Moreover, choose $n_{2}>n_{1}$ such that $\left|\beta_{k}\right|>\beta_{n_{1}}$ for all $k \geq n_{2}$. Now let $n \geq n_{2}$ be such that $\beta_{n}>0$. Then, for $k \geq n_{0}$,

$$
\begin{aligned}
\left|\beta_{k}-\beta_{n}\right| & =\left|\alpha_{k}-\alpha_{n}+\gamma_{k}-\gamma_{n}\right| \geq\left|\alpha_{k}-\alpha_{n}\right|-\left|\gamma_{k}\right|-\left|\gamma_{n}\right| \\
& \geq\left|\alpha_{k}-\alpha_{n}\right|-\frac{1}{4} s_{\alpha}(k)-\frac{1}{4} s_{\alpha}(n) \\
& \geq\left|\alpha_{k}-\alpha_{n}\right|-\frac{1}{4}\left|\alpha_{n}-\alpha_{k}\right|-\frac{1}{4}\left|\alpha_{k}-\alpha_{n}\right|=\frac{1}{2}\left|\alpha_{k}-\alpha_{n}\right|,
\end{aligned}
$$

and hence

$$
s_{\beta}(n)=\min \left\{\left|\beta_{k}-\beta_{n}\right|: k \geq n_{0}, k \neq n\right\} \geq \frac{1}{2} s_{\alpha}(n) .
$$

The proof for negative $\beta_{n}$ is similar and therefore $s_{\alpha}(n) \lesssim s_{\beta}(n), n \rightarrow \infty$. This relation also implies that $\lim _{n \rightarrow \infty} \frac{\left|\gamma_{n}\right|}{s_{\beta}(n)}=0$. With this property one can prove in the same way as above that $s_{\alpha}(n) \geq \frac{1}{2} s_{\beta}(n)$ for all sufficiently large indices $n$. Together, thus $s_{\beta}(n) \asymp s_{\alpha}(n)$.

Now it is clear that the condition (3.3) also holds for the sequence $\beta$ and the perturbation $-\gamma$. Again using that, for all sufficiently large $n$, the points $\alpha_{n}$ and $\beta_{n}$ have the same sign, we can conclude that (3.27) holds with $\beta$ and $-\gamma$ in place of $\alpha$ and $\gamma$.

Next, we turn to the relation between $r_{\alpha}(\rho, n)$ and $r_{\beta}(\rho, n)$. Let $\rho^{\prime} \in(1, \rho)$, and choose $n_{0} \in \mathbb{N}$ such that

$$
\left(\frac{\rho^{\prime}}{\rho}\right)^{\frac{1}{2}} \leq \frac{\beta_{k}}{\alpha_{k}} \leq\left(\frac{\rho}{\rho^{\prime}}\right)^{\frac{1}{2}}, \quad k \geq n_{0} .
$$

Moreover, let $n_{1} \geq n_{0}$ be such that $\frac{1}{\rho^{\prime}}\left|\beta_{n}\right| \geq \max _{l \leq n_{0}}\left|\beta_{l}\right|$ for $n \geq n_{1}$. Now fix $n \geq n_{1}$. If $k \in \mathbb{N}$ is such that $\frac{\beta_{k}}{\beta_{n}} \in\left(\frac{1}{\rho^{\prime}}, \rho^{\prime}\right)$, then $k \geq n_{0}$ and therefore

$$
\frac{\alpha_{k}}{\alpha_{n}}=\frac{\alpha_{k}}{\beta_{k}} \cdot \frac{\beta_{n}}{\alpha_{n}} \cdot \frac{\beta_{k}}{\beta_{n}}\left\{\begin{array}{l}
\leq\left(\frac{\rho^{\prime}}{\rho}\right)^{-\frac{1}{2}}\left(\frac{\rho}{\rho^{\prime}}\right)^{\frac{1}{2}} \rho^{\prime}=\rho, \\
\geq\left(\frac{\rho}{\rho^{\prime}}\right)^{-\frac{1}{2}}\left(\frac{\rho^{\prime}}{\rho}\right)^{\frac{1}{2}} \frac{1}{\rho^{\prime}}=\frac{1}{\rho} .
\end{array}\right.
$$

Hence

$$
\left\{k \in \mathbb{N}: \frac{\beta_{k}}{\beta_{n}} \in\left(\frac{1}{\rho^{\prime}}, \rho^{\prime}\right)\right\} \subseteq\left\{k \in \mathbb{N}: \frac{\alpha_{k}}{\alpha_{n}} \in\left(\frac{1}{\rho}, \rho\right)\right\}
$$

and this gives $r_{\beta}\left(\rho^{\prime}, n\right) \leq r_{\alpha}(\rho, n)$. Together with the already proved fact about separations, it follows that (3.4) holds for $\beta$ and $-\gamma$. 
Theorem 3.19 is not symmetric in $\alpha$ and $\beta$, but this is only a matter formulation. In fact, we can restate Theorem 3.19 as follows.

3.24 Theorem. Let $\alpha, \beta$ and $\Lambda$ be given as in Theorem 3.3. Assume that the following condition is satisfied.

(F) The sequence $\alpha$ contains infinitely many positive terms if and only if $\beta$ does. In this case there exists a growth function $\lambda^{+}$such that

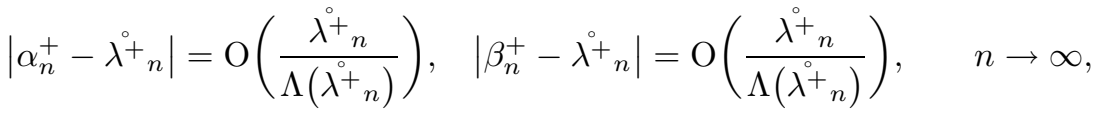

$$
\begin{aligned}
& \left|\alpha_{n}^{+}-\dot{\lambda}^{+}{ }_{n}\right|=\mathrm{O}\left(\frac{\lambda^{\circ}{ }_{n}}{n \log n}\right), \quad\left|\beta_{n}^{+}-\dot{\lambda}^{\circ}{ }_{n}\right|=\mathrm{O}\left(\frac{\lambda^{\circ}{ }_{n}}{n \log n}\right), \quad n \rightarrow \infty .
\end{aligned}
$$

If $\alpha$ contains infinitely many negative terms, the analogous statement holds.

Then (3.5) holds.

We decided to present the 'asymmetric formulation' of Theorem 3.19 as the principle formulation in order to emphasize that strong regularity is assumed.

\section{A selection of applications}

\subsection{The inverse spectral problem for a string}

A string is a pair $\mathrm{S}[L, m]$ that consists of a number $L, 0<L \leq \infty$, and a nonnegative and non-decreasing function $m$ defined on $[0, L)$. The string $\mathrm{S}[L, m]$ gives rise to an operator model, namely the Kreln-Feller differential operator $-D_{m} D_{x}$ acting in the space $L^{2}(d m)$. The eigenvalue equation for one of its self-adjoint realizations can be written in integral form as

$$
\left\{\begin{array}{l}
f(x)-f(0)+z \int_{[0, x]}(x-y) f(y) d m(y)=0, \quad x \in(0, L), \\
f^{\prime}(0-)=0,
\end{array}\right.
$$

where $z \in \mathbb{C}$ is the eigenvalue parameter; if the limit circle case prevails at $L$, i.e. if $\int_{[0, L)} x^{2} d m(x)<\infty$, then a boundary condition is also needed at $L$. The operator $-D_{m} D_{x}$ arises when Fourier's method is applied to the partial differential equation

$$
\frac{\partial}{\partial m(s)}\left(\frac{\partial v(s, t)}{\partial s}\right)-\frac{\partial^{2}}{\partial t^{2}} v(s, t)=0
$$

describing the vibrations of an inhomogeneous string with mass distribution $m$ and a free left endpoint.

The spectrum of the Krĕn-Feller operator $-D_{m} D_{x}$ is fully described by one analytic function: the principle Titchmarsh-Weyl coefficient $q_{\mathrm{S}}$ associated with the string $\mathrm{S}=\mathrm{S}[\mathrm{L}, \mathrm{m}]$; see, e.g. $[\mathrm{KaKr}]$. This function belongs to the Stieltjes class $\mathcal{S}$, i.e. $q_{\mathrm{S}}$ is analytic in the region $\mathbb{C} \backslash[0, \infty)$, has non-negative imaginary 
part throughout the upper half-plane, and takes non-negative values along the negative real half-line. A Fourier transform can be constructed which maps $-D_{m} D_{x}$ to the multiplication operator by the independent variable in $L^{2}\left(\mu_{\mathrm{S}}\right)$, where $\mu_{\mathrm{S}}$ is the measure in the representation of $q_{\mathrm{S}}$ as a Cauchy integral:

$$
q_{\mathrm{S}}(z)=a+\int_{\mathbb{R}} \frac{d \mu_{\mathrm{S}}(t)}{t-z} .
$$

The cornerstones of the spectral theory of strings were established by M. G. Kre ̌n in the early $1950 \mathrm{~s}$, see $[\mathrm{Kr} 3]$ or $[\mathrm{KaKr} \text {, Theorem } 11.2]^{8}$; a presentation from a slightly different viewpoint can be found in [DK]. Most notably, an inverse spectral theorem was proved. For this we have to normalize $L$ and $m$, i.e. we assume that $m(0)=0$, that $m$ is left continuous and that $m(x)<\sup _{t \in[0, L)} m(t)$ for all $x \in[0, L)$. Then the inverse spectral theorem reads as follows.

For each function $q \in \mathcal{S}$, there exists a unique string $\mathrm{S}[L, m]$ such that $q$ is the principle Titchmarsh-Weyl coefficient of $\mathrm{S}[L, m]$.

The number $a$ in (4.1) gives the length of a massless initial section of the string, i.e. $a=\sup \{x: m(x)=0\}$. In the following we assume that $a=0$, i.e. that $m(x)>0$ for $x>0$.

A string $\mathrm{S}[L, m]$ is called regular if

$$
L<\infty \quad \text { and } \quad m(L):=\lim _{x \nearrow L} m(x)<\infty,
$$

and singular otherwise ${ }^{9}$. Thinking of direct and inverse spectral relations, the problem arises to describe the totality $\mathcal{S}_{\text {reg }}$ of all Stieltjes class functions that are principle Titchmarsh-Weyl coefficients of regular strings. The solution of this problem is known. It follows from [Kr2], see also $\left[\mathrm{KaKr}, 11.11^{\circ}\right]^{10}$.

4.1 Theorem $([\mathrm{Kr} 2])$. Let $\mu$ be a positive measure with $\operatorname{supp} \mu \subseteq(0, \infty)$ and $\int_{\mathbb{R}} \frac{d \mu(t)}{1+t}<\infty$. Consider the Cauchy transform $q_{\mu}(z):=\int_{\mathbb{R}} \frac{d \mu(t)}{t-z}$ of $\mu$, and let $\mathrm{S}[L, m]$ be the string whose principle Titchmarsh-Weyl coefficient is equal to $q_{\mu}$. Then $\mathrm{S}[L, m]$ is regular if and only if

(i) the measure $\mu$ is discrete, say $\mu=\sum_{n=1}^{N} \sigma_{n} \delta_{\alpha_{n}}$ with $N \in \mathbb{N} \cup\{\infty\}, \sigma_{n}>0$ and $0<\alpha_{1}<\alpha_{2}<\ldots$;

(ii) the limit $\lim _{n \rightarrow \infty} \frac{n}{\sqrt{\alpha_{n}}}$ exists and is finite (we tacitly understand this limit as 0 if $N$ is finite);

(iii) we have

$$
\sum_{n=1}^{N} \frac{1}{\alpha_{n}^{2}\left(P_{\alpha}^{\prime}\left(\alpha_{n}\right)\right)^{2} \sigma_{n}}<\infty
$$

It is clear that a sequence $\alpha$ satisfying $(i)-(i i i)$ belongs to $\mathbb{S}$ introduced in Definition 3.1. Applying Theorem 3.3 (or Theorems 3.18, 3.19) we immediately obtain a stability result for the class $\mathcal{S}_{\text {reg }}$, which says that sufficiently small shifts of poles do not lead out of the class $\mathcal{S}_{\text {reg. }}$. The precise formulation reads as follows.

\footnotetext{
${ }^{8}$ For the reason of physical interpretation, in $[\mathrm{KaKr}]$ the principle Titchmarsh-Weyl coefficient is called 'coefficient of dynamic compliance'.

${ }^{9} \mathrm{In}[\mathrm{DK}]$ the maybe more descriptive terminology 'short' and 'long' is used.

${ }^{10}$ For simplicity we restrict ourselves to the case that $0 \notin \operatorname{supp} \mu$.
} 
4.2 Theorem. Let $\mu$ be a discrete measure, $\mu=\sum_{n=1}^{\infty} \sigma_{n} \delta_{\alpha_{n}}$, with

$$
\begin{aligned}
& 0<\alpha_{1}<\alpha_{2}<\ldots, \quad \lim _{n \rightarrow \infty} \frac{n}{\sqrt{\alpha_{n}}} \in[0, \infty), \\
& \sigma_{n}>0, \quad \sum_{n=1}^{\infty} \frac{\sigma_{n}}{\alpha_{n}}<\infty,
\end{aligned}
$$

and set $q_{\mu}(z):=\int_{\mathbb{R}} \frac{d \mu(t)}{t-z}$.

Let $\gamma=\left(\gamma_{n}\right)_{n \in \mathbb{N}}$ be a sequence of real numbers such that the points $\beta_{n}:=$ $\alpha_{n}+\gamma_{n}$ are all positive and pairwise distinct, let $\tau_{n}, n \in \mathbb{N}$, be positive real numbers, and set

$$
\nu=\sum_{n=1}^{\infty} \tau_{n} \delta_{\beta_{n}}, \quad q_{\nu}(z):=\int_{\mathbb{R}} \frac{d \nu(t)}{t-z} .
$$

If the hypotheses of one of Theorems 3.3, 3.18, 3.19 are fulfilled and $\tau_{n} \asymp \sigma_{n}$, then

$$
q_{\mu} \in \mathcal{S}_{\text {reg }} \quad \Longleftrightarrow \quad q_{\nu} \in \mathcal{S}_{\text {reg }} .
$$

\subsection{The Krel̆n class of entire functions}

Let $f$ be an entire function with $f(0)=1$, and denote its sequence of zeros by $\alpha=\left(\alpha_{n}\right)_{n \in \mathbb{N}}$, which are assumed to be simple. Then $f$ is said to belong to the Kreı̆n class $\mathbb{K}$ if

(Kr1) $\sum_{n \in \mathbb{N}}\left|\operatorname{Im} \frac{1}{\alpha_{n}}\right|<\infty$;

(Kr2) there exists a number $l \in \mathbb{N}$ such that

$$
\sum_{n \in \mathbb{N}} \frac{1}{\left|\alpha_{n}\right|^{l}\left|f^{\prime}\left(\alpha_{n}\right)\right|}<\infty,
$$

and, on the domain $\mathbb{C} \backslash\left\{\alpha_{n}: n \in \mathbb{N}\right\}$, the function $\frac{1}{f}$ is represented as

$$
\frac{1}{f(z)}=p(z)+\sum_{n \in \mathbb{N}} \frac{1}{f^{\prime}\left(\alpha_{n}\right)}\left(\frac{1}{z-\alpha_{n}}+\frac{1}{\alpha_{n}}+\ldots+\frac{z^{l-2}}{\alpha_{n}^{l-1}}\right)
$$

with some polynomial $p$.

This class was first studied by M. G. Kreln in the 1940s. It appears, e.g. in the context of extension theory of symmetric operators in a Hilbert space, cf. [Kr1]; for properties of functions in $\mathbb{K}$ see, e.g. [L1, Section V.6]. M. G. Krel̆n proved that every function from the class $\mathbb{K}$ is of exponential type and has completely regular growth; see, e.g. [L1, Theorem V.13].

Often functions that have only real zeros are of particular interest. We therefore introduce the following subclasses.

4.3 Definition. Let $l \in \mathbb{N}$. We say that a function $f$ belongs to the class $\mathbb{K}_{l}$ if

(i) $f$ is entire, takes real values along the real axis, satisfies $f(0)=1$ and has only real and simple zeros which are all non-zero; 
(ii) the condition (4.3) and the representation (4.4) hold for the number $l$ and with some polynomial $p$ of degree at most $l-2$; here we understand that $p$, as well as the regularising terms in the sum, are not present when $l=1$.

Note that $\mathbb{K}_{l} \subseteq \mathbb{K}_{l+1} \subseteq \mathbb{K}, l \in \mathbb{N}$, and $\bigcup_{l \in \mathbb{N}} \mathbb{K}_{l}$ is the set of all real $f \in \mathbb{K}$ with only real zeros.

It follows from [L1, Theorems V.11 and V.13] that every function $f \in$ $\bigcup_{l \in \mathbb{N}} \mathbb{K}_{l}$ is a canonical product and its zeros build a sequence belonging to the class $\mathbb{S}$, i.e. $f=P_{\alpha}$ with $\alpha \in \mathbb{S}$. As an application of our main theorems we prove the following perturbation result for the classes $\mathbb{K}_{l}$.

4.4 Theorem. Let $\alpha, \beta \in \mathbb{S}$, and let $l \in \mathbb{N}$. If the hypotheses of one of Theorems $3.3,3.18,3.19$ are fulfilled, then

$$
P_{\alpha} \in \mathbb{K}_{l} \quad \Longleftrightarrow \quad P_{\beta} \in \mathbb{K}_{l} .
$$

In order to establish the theorem, we use the following fact. Since an explicit reference is not known to us, we provide its proof.

4.5 Lemma. Let $l \in \mathbb{N}$, let $\alpha \in \mathbb{S}$ and assume that

$$
\sum_{n \in \mathbb{N}} \frac{1}{\left|\alpha_{n}\right|^{l}\left|P_{\alpha}^{\prime}\left(\alpha_{n}\right)\right|}<\infty
$$

Then $P_{\alpha} \in \mathbb{K}_{l}$.

Proof. It is clear that $P_{\alpha}$ satisfies $(i)$. By [LW1, Lemma 5.5], convergence of the series (4.5) implies that $P_{\alpha}$ is of bounded type in the upper and lower halfplanes $\mathbb{C}^{+}$and $\mathbb{C}^{-}$; for the definition of functions of bounded type see, e.g. [dB, Section 8].

Consider the function

$$
\begin{aligned}
g(z) & :=\sum_{k \in \mathbb{N}} \frac{1}{P_{\alpha}^{\prime}\left(\alpha_{k}\right)}\left(\frac{1}{z-\alpha_{k}}+\frac{1}{\alpha_{k}}+\ldots+\frac{z^{l-2}}{\alpha_{k}^{l-1}}\right) \\
& =z^{l-1} \sum_{k \in \mathbb{N}} \frac{1}{P_{\alpha}^{\prime}\left(\alpha_{k}\right)\left(z-\alpha_{k}\right) \alpha_{k}^{l-1}} .
\end{aligned}
$$

Due to (4.5), this series converges absolutely and locally uniformly on the set $\mathbb{C} \backslash\left\{\alpha_{n}: n \in \mathbb{N}\right\}$, and thus represents an analytic function on this domain. At the points $\alpha_{n}$ it has simple poles with residua $\frac{1}{P_{\alpha}^{\prime}\left(\alpha_{n}\right)}$. The function $g$ can be written as

$$
g(z)=z^{l-1} \int_{\mathbb{R}} \frac{d \mu}{z-t},
$$

where $\mu$ is the discrete complex measure having point masses at the points $\alpha_{k}$ with masses $\frac{1}{P_{\alpha}^{\prime}\left(\alpha_{k}\right) \alpha_{k}^{l-1}}$. Hence $g$ is of bounded type in both half-planes $\mathbb{C}^{+}$and $\mathbb{C}^{-}$.

We conclude that the difference

$$
h(z):=\frac{1}{P_{\alpha}(z)}-g(z)
$$


is an entire function which is of bounded type in $\mathbb{C}^{+}$and $\mathbb{C}^{-}$. By Kreln's theorem [RR, Theorems $6.17,6.18], h$ is of finite exponential type equal to the maximum of the mean types in $\mathbb{C}^{+}$and $\mathbb{C}^{-}$.

Since $y \mapsto\left|1-\frac{i y}{\alpha_{n}}\right|$ is increasing on $(0, \infty)$ and decreasing on $(-\infty, 0)$ and $\lim _{y \rightarrow \pm \infty}\left|1-\frac{i y}{\alpha_{n}}\right|=\infty$ for each $n \in \mathbb{N}$, we have $\lim _{y \rightarrow \pm \infty} \frac{1}{P_{\alpha}(i y)}=0$. Using dominated convergence, we see that $\lim _{y \rightarrow \pm \infty} \frac{1}{y^{1-1}} g(i y)=0$, and together thus

$$
\lim _{y \rightarrow \pm \infty} \frac{1}{y^{l-1}} h(i y)=0 .
$$

We conclude that $h$ is of minimal exponential type and, applying the PhragménLindelöf principle [Bo, Theorem 1.4.3], that $h$ is in fact a polynomial of degree at most $l-2$ (here we understand that $h$ vanishes identically if $l=1$ ). Thus $\frac{1}{f}$ is represented as required in (4.4), and we have shown that $f \in \mathbb{K}_{l}$.

Proof of Theorem 4.4. Assume that $P_{\alpha} \in \mathbb{K}_{l}$. Then the function $P_{\alpha}$ satisfies (4.5). Since $\lim _{n \rightarrow \infty} \frac{\alpha_{n}}{\beta_{n}}=1$ and the quotient $\left|\frac{P_{\alpha}^{\prime}\left(\alpha_{n}\right)}{P_{\beta}^{\prime}\left(\beta_{n}\right)}\right|$ is bounded from above and away from zero, also the function $P_{\beta}$ satisfies (4.5). Hence, we may apply Lemma 4.5 and conclude that $P_{\beta} \in \mathbb{K}_{l}$.

For the converse, regard $\alpha$ as a perturbation of $\beta$, cf. Proposition 3.23 and Theorem 3.24.

\subsection{The continuation problem for a positive definite func- tion}

A continuous function $f: \mathbb{R} \rightarrow \mathbb{C}$ is called positive definite if

(PD1) $f(-t)=\overline{f(t)}, \quad t \in \mathbb{R}$;

(PD2) the kernel $f(t-s)$ is positive semi-definite; this means that, for each choice of $n \in \mathbb{N}$ and $t_{1}, \ldots, t_{n} \in \mathbb{R}$, the quadratic form

$$
Q\left(\xi_{1}, \ldots, \xi_{n}\right)=\sum_{i, j=1}^{n} f\left(t_{i}-t_{j}\right) \xi_{i} \overline{\xi_{j}}
$$

is positive semi-definite.

By Bochner's theorem the set of all positive definite functions coincides with the set of all Fourier transforms of finite positive measures on the real line, i.e. a function $f$ is positive definite if and only if it can be represented as

$$
f(t)=\int_{\mathbb{R}} e^{-i t x} d \mu(x), \quad t \in \mathbb{R},
$$

with some finite positive measure $\mu$.

Let $f$ be a positive definite function, let $a>0$, and consider the restriction

$$
\varphi:=\left.f\right|_{[-2 a, 2 a]} .
$$

Then it may happen that there exist also other positive definite functions whose restriction to the interval $[-2 a, 2 a]$ equals $\varphi$. In fact, it is a classical result that either (I) or (II) holds: 
(I) $\quad f$ is the only positive definite function with $\left.f\right|_{[-2 a, 2 a]}=\varphi$.

(II) There exist infinitely many positive definite functions $\tilde{f}$ that satisfy $\left.\tilde{f}\right|_{[-2 a, 2 a]}=\varphi$.

A proof of this alternative and a parameterization of the set of all functions $\tilde{f}$ in case (II) can be given. One possible approach proceeds via operator theoretic methods; see, e.g. [GG, §3.2].

Assume that case (I) prevails for $f$ and $a$. When the requirement that the extension is positive definite is slightly weakened, it may happen that $\varphi$ can still be extended in infinitely many ways to the whole real line. To make this precise, we say that a continuous function $f: \mathbb{R} \rightarrow \mathbb{C}$ is Hermitian indefinite with negative index $\kappa \in \mathbb{N}$ if it satisfies (PD1) and

$\left(\mathbf{P D 2}_{\kappa}\right)$ the kernel $f(t-s)$ has $\kappa$ negative squares; this means that, for each choice of $n \in \mathbb{N}$ and $t_{1}, \ldots, t_{n} \in \mathbb{R}$, the quadratic form (4.6) has at most $\kappa$ negative squares, and, for some choice of $n, t_{1}, \ldots, t_{n}$, this upper bound is attained.

4.6 Definition. Let $f$ be a positive definite function, let $a>0$, and assume that case (I) prevails for $f$ and $a$. Then we set

$$
\Delta(f, a):=\inf \left\{\kappa \in \mathbb{N}: \exists \tilde{f} \text { with }(\mathrm{PD} 1),\left(\mathrm{PD} 2_{\kappa}\right) \text { s.t. }\left.\tilde{f}\right|_{[-2 a, 2 a]}=\varphi\right\} .
$$

Here the infimum of the empty set is understood as being equal to $\infty$.

4.7 Example. The function $f(t)$ that equals $1-|t|$ on $[-2,2]$ and is continued periodically to $\mathbb{R}$ is positive definite as can be seen from its Fourier series. For $a=1$, case (I) prevails and $\Delta(f, 1)=1$; see [LLS]. An extension of $\left.f\right|_{[-2,2]}$ with one negative square is, e.g. the function $\tilde{f}(t)=1-|t|, t \in \mathbb{R}$.

Consider $f$ and $a$ such that case (I) prevails, and let $\mu$ be the inverse Fourier transform of $f$. A characterization of ' $\Delta(f, a)<\infty$ ' in terms of $\mu$ can be given; in fact the actual value of $\Delta(f, a)$ can be computed, cf. [W, Proposition 6.11].

4.8 Theorem $([\mathrm{W}])$. Let $f$ be a positive definite function, let $a>0$, and assume that case (I) prevails for $f$ and a. Moreover, let $\mu$ be the inverse Fourier transform of $f$, i.e. $\mu$ is the positive finite measure with $f(t)=\int_{\mathbb{R}} e^{-i t x} d \mu(x)$. Then $\Delta(f, a)<\infty$ if and only if

(i) the measure $\mu$ is discrete, say $\mu=\sum_{n=1}^{N} \sigma_{n} \delta_{\alpha_{n}}$ with $N \in \mathbb{N} \cup\{\infty\}$ and $\sigma_{n}>0$

(ii) the sequence $\alpha=\left(\alpha_{n}\right)_{n \in \mathbb{N}}$ belongs to $\mathbb{S}$;

(iii) there exists a number $k \in \mathbb{N}$ such that

$$
\sum_{n=1}^{N} \frac{1}{\alpha_{n}^{2 k}\left(P_{\alpha}^{\prime}\left(\alpha_{n}\right)\right)^{2} \sigma_{n}}<\infty
$$

If $\Delta(f, a)<\infty$ and $k_{0}$ denotes the smallest natural number such that (4.7) holds, then

$$
\Delta(f, a)=k_{0}-1
$$


We invoke the present stability results.

4.9 Theorem. Let $f$ be a positive definite function, let $a>0$, and let $\mu$ be the inverse Fourier transform of $f$. Assume that case (I) prevails for $f$ and a, that $\mu$ is discrete, say $\mu=\sum_{n=1}^{\infty} \sigma_{n} \delta_{\alpha_{n}}$ with $\sigma_{n}>0$, and that $\alpha \in \mathbb{S}$. Let $\beta \in \mathbb{S}$ and $\tau_{n}>0$, and consider

$$
\nu:=\sum_{n \in \mathbb{N}} \tau_{n} \delta_{\beta_{n}}, \quad g(t):=\int_{\mathbb{R}} e^{-i t x} d \nu(x) .
$$

If the hypotheses of one of Theorems 3.3, 3.18, 3.19 are fulfilled, $\tau_{n} \asymp \sigma_{n}$ and case (I) prevails for $g$ and $a$, then

$$
\Delta(g, a)=\Delta(f, a) .
$$

4.10 Remark. It is an open problem whether the hypotheses of Theorem 4.9 already imply that case (I) prevails for $g$ and $a$.

\section{References}

[Bo] R. BoAs: Entire Functions, Academic Press, New York, 1954.

[dB] L. De Branges: Hilbert Spaces of Entire Functions, Prentice-Hall, London, 1968.

[DK] H. Dym, H. McKean: Gaussian Processes, Function Theory, and the Inverse Spectral Problem, Academic Press, New York, 1976.

[GG] M.L. Gorbachuk, V.I. Gorbachuk: M.G. Krĕ̌n's Lectures on Entire Operators, Oper. Theory Adv. Appl. 97, Birkhäuser Verlag, Basel, 1997.

[KaKr] I.S. KAC, M.G. KREĬN: On the spectral functions of a string, in F.V.Atkinson, Discrete and Continuous Boundary Problems (Russian translation), Mir, Moscow, 1968, 648-737 (Addition II). English translation: Amer. Math. Soc. Transl. (2) 103 (1974), 19-102.

[Kr1] M.G. KREǏN: The fundamental propositions of the theory of representations of Hermitian operators with deficiency index $(m, m)$. (Russian), Ukrain. Mat. Zurnal 1 (1949), no. 2, 3-66.

[Kr2] M.G. KREINN: Determination of the density of a nonhomogeneous symmetric cord by its frequency spectrum. (Russian), Doklady Akad. Nauk SSSR (N.S.) 76 (1951), 345-348.

[Kr3] M.G. KREǏN: On a generalization of investigations of Stieltjes. (Russian), Doklady Akad. Nauk SSSR (N.S.) 87 (1952), 881-884.

[LLS] H. LANGer, M. LANGer, Z. SASvÁRI: Continuations of Hermitian indefinite functions and corresponding canonical systems: an example, Methods Funct. Anal. Topology 10 (2004), 39-53.

[LW1] M. LANGer, H. WoraceK: A characterization of intermediate Weyl coefficients, Monatsh. Math. 135 (2002), 137-155.

[LW2] M. Langer, H. WoraceK: Stability of N-extremal measures, submitted, Preprint: ASC Report 05/2013, Vienna University of Technology, 2013, http://www.asc.tuwien.ac.at/preprint/2013/asc05×2013.pdf.

[LW3] M. LAnger, H. Woracek: A Pontryagin space approach to the index of determinacy of a measure, manuscript in preparation. 
[LG] P. Lelong, L. Gruman: Entire Functions of Several Variables, SpringerVerlag, Berlin, 1986.

[L1] B.J. Levin: Distribution of Zeros of Entire Functions (Russian), Gosudarstv. Izdat. Tehn.-Teor. Lit., Moscow, 1956. English translation: Translations of Mathematical Monographs, vol. 5, Amer. Math. Soc., Providence, R.I., 1964.

[L2] B.YA. Levin: Lectures on Entire Functions, Translated from the Russian manuscript by V.Tkachenko. Translations of Mathematical Monographs, vol. 150. Amer. Math. Soc., Providence, RI, 1996.

[RR] M. Rosenblum, J. Rovnyak: Topics in Hardy Classes and Univalent Functions, Birkhäuser Verlag, Basel, 1994.

[Ru] L.A. Rubel: Entire and Meromorphic Functions, Springer-Verlag, New York, 1996.

[S] E. Seneta: Regularly Varying Functions, Lecture Notes in Mathematics, vol. 508, Springer, Berlin-Heidelberg-New York, 1976.

[W] H. WORACEK: Existence of zerofree functions $N$-associated to a de Branges Pontryagin space, Monatsh. Math. 162 (2011), 453-506.

M. Langer

Department of Mathematics and Statistics

University of Strathclyde

26 Richmond Street

Glasgow G1 1XH

UNITED KINGDOM

email: m.langer@strath.ac.uk

H. Woracek

Institut für Analysis und Scientific Computing

Technische Universität Wien

Wiedner Hauptstraße. 8-10/101

1040 Wien

AUSTRIA

email: harald.woracek@tuwien.ac.at 